\title{
INFLUENCE DE LA LIAISON ENTRE CHAMBRE D'ÉQUILIBRE ET CANAL D'AMENÉE D'UNE USINE HYDRO-ELECTRIQUE SUR LE RÉGIME DES SURPRESSIONS - ROLE DE L'ETRANGLEMENT
}

\author{
THE INFLUENCE ON A HYDRO-ELECTRIC PLANT OF CONNECTING THE SURGE \\ TANK TO THE HEAD-RACE ROLE PLAYED BY THE SURGE TANK THROAT
}

\author{
Das' 1.. ESCANDE \\ Ingénieur 1. E.T. \\ Professeur à !a Faculté des Sciences \\ Directeur de l'Ecole Nationale Supérieure d'Electrotechnique et d'Hydraulique de Toulouse
}

English synopsis p. 436

\section{INTRODUCTION}

Dans cette étude, nous nous proposons de préciser l'influence que peut avoir la liaison entre chambre d'équilibre et canal d'amenée d'une usine hydro-électrique sur le régime des surpressions intervenant dans le système canal d'amenée-conduite forcée de cette usine, en régime transitoire.

Nous examinons de façon plus particulière le cas des chambres à étranglement inférieur, ce dispositif ayant donné lieu parfois à des critiques injustifiées.

La méthode graphique de M. BERGERON permet d'analyser les phénomènes, dans tous les cas, et de montrer que, pour toutes les manœuvres susceptibles d'intervenir dans une usine, la présence de l'étranglement n'aggrave en rien le régime des surpressions.

Poussant plus loin cette analyse, nous envisageons le cas de fermetures accidentelles du distributeur extrêmement brèves et nous donnons une formule permettant de calculer la surpression maximum existant dans ce cas, sous l'étranglement.

Nous pensons que ce travail, inspiré par les questions qui nous furent posées, d'une part par les Etablissements SCHNEIDER, en ce qui concerne les principes généraux, d'autre part par M. PARISOT, directeur à l'Energie Industrielle, à propos de l'usine des Brévières, contribuera à dissiper la méfiance qu'inspire encore à certains ingénieurs le dispositif d'étranglement.
CHAPITRE PREMIER

\section{PROBLEME PRÉLIMINAIRE TRANSMISSION ET REFLEXION D'UNE ONDE A UNE BIFURCATION DE CONDUITES}

Nous rappelons certains résultats classiques: considérons tro:s conduites (fig. 1): C, $\mathrm{C}^{\prime}, \mathrm{C}^{\prime \prime}$ correspondant: $C$ à la conduite forcée, $C^{\prime}$ à la liaison avec la chambre d'équilibre, C" au canal d'amenée; soient S, S', S" leurs sections, a, $a^{\prime}, a^{\prime \prime}$ les vitesses de propagation des ondes, $\beta=\frac{a}{s}$, $\Theta^{\prime}=\frac{a^{\prime}}{S^{\prime}}, \quad \Theta^{\prime \prime}=\frac{a^{\prime \prime}}{S^{\prime \prime}}$ les caractéristiques.

Soit, dans le régime permanent initial, $v_{0}, v_{0}{ }^{\prime}$ $v_{0}, "$ les vitesses telles que l'on ait, par continuité:

$$
S v_{0}=S^{\prime} v_{11}^{\prime}+S^{\prime \prime} v_{.11}^{\prime \prime}
$$

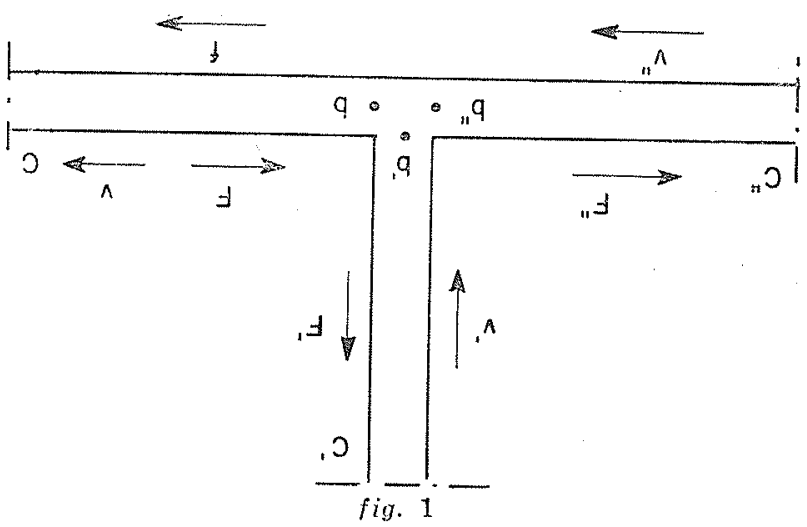


Supposons qu'une manœuvre instantanée des turbines provoque la formation, dans la conduite $C$, d'une onde à front vertical $F$. Celle-ci, à son arrivée à la bifurcation $b, b^{\prime}, b^{\prime \prime}$ engendre une onde $F^{\prime}$ transmise dans $C^{\prime}$, une onde $F^{\prime \prime}$ transmise dans $C^{\prime \prime}$ et une onde $f$ réfléchie dans $C$.

La pression ayant la même valeur aux trois points confondus b, b', $b^{\prime \prime}$, c'est-à-dire qu'elle soit évaluée en considérant ce point comme appartenant à l'une ou à l'autre des trois conduites, on doit avoir:

$$
y_{0}+F+f=y_{0}+F^{\prime}=y_{0}+F^{\prime \prime}
$$

ou :

$$
F^{\prime}=F^{\prime \prime}=F+f
$$

La continuité exige, par ailleurs, que l'on ait :

$$
S\left[v_{0}-\frac{g}{a}(F-f)\right]=S^{\prime}\left[v_{0}^{\prime}-\frac{g}{a^{\prime}} F^{\prime}\right]+S^{\prime \prime}\left[v_{0}^{\prime \prime}-\frac{g}{a} F^{\prime \prime}\right]
$$

ou, compte tenu de (1):

$$
\frac{F-f}{\beta}=\frac{F^{\prime}}{\beta^{\prime}}+\frac{F^{\prime \prime}}{\beta^{\prime \prime}}
$$

On tire immédiatement des équations (2) et (3) les relations classiques:

$$
\begin{aligned}
& F^{\prime}=F^{\prime \prime}=F \times \frac{2 \beta^{\prime} \beta^{\prime \prime}}{\beta \beta^{\prime}+\beta^{\prime \prime} \beta^{\prime \prime}+\beta^{\prime \prime} \beta} \\
& f=F \times \frac{\beta^{\prime} \beta^{\prime \prime}-\beta\left(\beta^{\prime}+\beta^{\prime \prime}\right)}{\beta \beta^{\prime}+\beta^{\prime} \beta^{\prime \prime}+\beta^{\prime \prime} \beta}
\end{aligned}
$$

\section{CHAPITRE II}

\section{TRANSMISSION AU CANAL D'AMENÉE D'UNE ONDE DE SURPRESSION A FRONT VERTICAL PROVENANT DE LA CONDUITE}

Le front de l'onde F est, par hypothèse, rigoureusement vertical.

A) Prenons, tout d'abord, le cas où le canal d'amenée et la conduite forcée débouchent tous deux directement dans la chambre d'équilibre. et raisonnons sur les données suivantes:

$\mathrm{s}=3,46 \mathrm{~m}^{2}, \quad a=1.000 \mathrm{~m} / \mathrm{sec}, \quad \beta=289$

$\mathrm{s}^{\prime}=50 \quad \mathrm{~m}^{2}, \quad \mathrm{a}^{\prime}=1.300 \mathrm{~m} / \mathrm{sec}, \quad \mathrm{g}^{\prime}=26$

$\mathrm{S}^{\prime \prime}=4,50 \mathrm{~m}^{2}, \quad a^{\prime \prime}=1.300 \mathrm{~m} / \mathrm{sec} ., \quad \beta^{\prime \prime}=289$
Les relations (4) et (5) donnent:

$$
F^{\prime}=F^{\prime \prime}=0,152 F ; \quad f=-0,848 F
$$

On voit que, malgré le débouché direct du canal dans la chambre d'équilibre, une onde à front raide est transmise au canal d'amenée dans la proportion de $15 \%$ environ, cependant que l'onde de dépression réfléchie dans la conduite est égale à $85 \%$ de l'onde de surpression incidente.

La section de la chambre devrait être infinie $\left(\beta^{\prime}=0\right)$ pour que l'on ait :

$$
F^{\prime}=0=F^{\prime \prime}, \quad f=-F
$$

B) Supposons maintenant que $C^{\prime}$ soit un tronçon de liaison avec la chambre d'équilibre, de section $\mathrm{S}^{\prime}=\mathrm{S}^{\prime \prime}=4,50 \mathrm{~m}^{\prime \prime}$, de vitesse d'onde $\mathrm{a}^{\prime}=1.300 \mathrm{~m} . / \mathrm{sec}$.

On a, dans ce cas:

$$
F^{\prime}=F^{\prime \prime}=0,666 \mathrm{~F} ; \quad f=-0,333 \mathrm{~F}
$$

L'onde transmise au canal est égale aux deux tiers de l'onde provenant de la conduite.

Faisons intervenir la longueur du tronçon $\mathrm{C}^{\prime}$ et supposons la égale à $6,50 \mathrm{~m}$. A son arrivée à la section d'épanouissement, supposée infiniment grande, l'onde $F$ ' subit une réflexion totale avec changement de signe et cette onde de dépression revient au point $b^{\prime}$ à un instant postérieur de 0,01 seconde à celui de l'arrivée de $F$ en $b$ : à partir de cet instant, par le jeu des réflexions successives dans le tronçon $C^{\prime}$, les ondes $F, F^{\prime}$ : deviennent rapidement nulles suivant un mécanisme qui sera analysé plus loin de façon détaillée (fig. 15).

II n'en résulte pas moins qu'une onde de surpression $F^{\prime \prime}=0,666 \mathrm{~F}$, de longueur $1.300 \times 0.01=13 \mathrm{~m}$. remonte le canal d'amenée sur toute sa longueur.

C) Considérons enfin une chambre d'équilibre de section infiniment grande munie d'un orifice d'étranglement inférieur que nous assimilerons à un conduit $C^{\prime}$, de longueur $L^{\prime}=1,30 \mathrm{~m}$, de section $\mathrm{S}^{\prime}=1 \mathrm{~m}^{2}$, de vitesse d'onde $a^{\prime}=1.300 \mathrm{~m} . / \mathrm{sec}$, de caractéristique $\beta^{\prime}=1.300$; on $a$, dans ce cas:

$$
F^{\prime}=F^{\prime \prime}=0,90 F ; \quad f=-0,10 F
$$

L'onde transmise au canal d'amenée n'est supérieure que de $30 \%$ à celle qui correspond 
au cas d'une liaison ordinaire. De plus, la durée de l'aller retour d'onde dans l'étranglement, n'est plus que de 0,002 sec., de telle sorte que la longueur de la tête d'onde transmise conservant la valeur maximum ci-dessus calculée, n'est plus que : $1.300 \times 0,002=2,60 \mathrm{~m}$., l'extinction de l'onde étant elle-même plus rapide que dans le cas précédent, par suite de la fréquence plus élevée des réflexions d'onde aux extrémités de l'étranglement.

D) En pratique, les ondes ne sont jamais à front vertical: nous avons tenu cependant à envisager ce cas théorique extrême, afin de montrer que si l'étranglement ne peut alors éviter la transmission d'une onde de surpression notable au canal d'amenée, les conditions sont sensiblement aussi dangereuses lorsqu'en l'absence d'étranglement la chambre d'équilibre est reliée au canal d'amenée par uñ conduit de section comparable à celle de ce canal.
CHAPITRE III

\section{TRANSMISSION AU CANAL D'AMENÉE DES ONDES A FRONT TRÈS INCLINÉ PROVENANT DE LA CONDUITE DANS LE CAS OU LA LIAISON $C$ A UNE GRANDE LONGUEUR PROBLÈME DE L'USINE DES BRÉVIÈRES}

En pratique, les manceuvres normales des turbines ne donnent naissance qu'à des ondes à front très incliné. Nous allons voir que celles-ci ne peuvent se transmettre partiellement au canal d'amenée que dans certains cas exceptionnels, tels que celui de l'usine des Brévières correspondant à une conduite de liaison de grande longueur. La remarquable méthode graphique de M. BERGERON permet d'étudier sans difficultés le régime des surpressions qui s'établissent dans un tel système (fig. 2), que nous supposons caractérisé par les données suivantes:

$$
\begin{aligned}
& \text { - Conduite } A_{b} . \quad L=350 \mathrm{~m} ., \quad S=9,82 \mathrm{~m}^{2} \text {, } \\
& a=1.000 \mathrm{~m} . / \mathrm{sec} ., \quad \sigma=-\frac{2 \mathrm{~L}}{\mathrm{a}}=0,70 \mathrm{sec} \text {. } \\
& \text { a } a \\
& \text { - Liaison } B^{\prime} b^{\prime}: \quad L^{\prime}=230 \mathrm{~m} ., \quad S^{\prime}=12.57 \mathrm{~m}^{2} \text {, } \\
& \text { (sans étranglement) } \begin{aligned}
a^{\prime} & =1.320 \mathrm{~m} . / \mathrm{sec} .,
\end{aligned} \quad \mathrm{o}^{\prime}=\frac{2 \mathrm{~L}^{\prime}}{\mathrm{a}^{\prime}}=0,35 \mathrm{sec}=\frac{\theta}{2} \\
& \text { - Canal B b": } \quad L^{\prime}=920 \mathrm{~m} \text {., } \\
& a^{\prime \prime}=1.320 \mathrm{~m} . / \mathrm{sec} ., \quad S^{\prime \prime}=11,045 \mathrm{~m}^{2} \text {, } \\
& \frac{\mathrm{a}^{\prime \prime}}{\mathrm{gS} \cdot \mathrm{\prime}}=12,19 \\
& 6^{\prime \prime}=\frac{2 L^{\prime \prime}}{a^{\prime \prime}}=1,4 \mathrm{sec}=20
\end{aligned}
$$

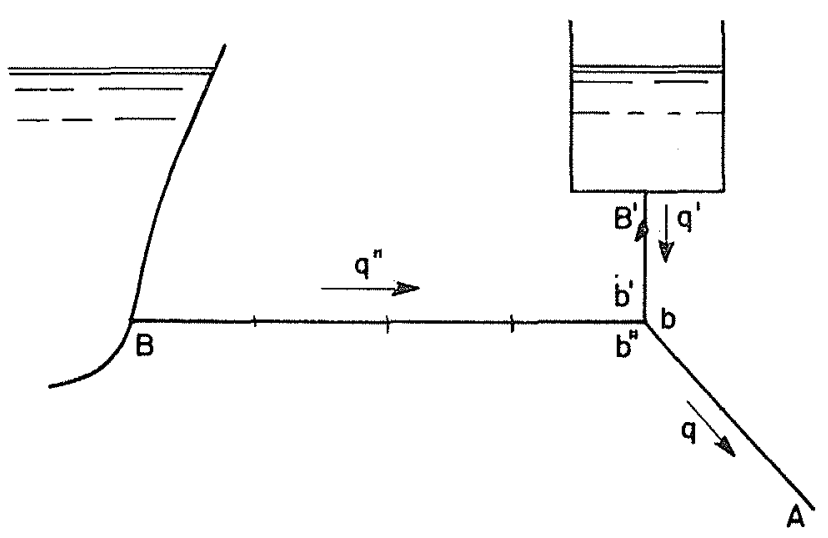

fill. ?

\section{Conventions.}

En première approximation, pour l'étude du coup de bélier d'onde, nous ne tenons pas compte du déplacement du niveau dans la chambre d'équilibre et nous négligeons toutes les pertes de charge, y compris celle qui se produit à la traversée de l'étranglement.

Nous adoptons comme unité de temps provisoire, pour la construction graphique:

$$
\theta^{\prime}=\frac{\theta}{2}=\frac{\theta^{\prime \prime}}{4}
$$

de telle sorte qu'une onde va de $A$ en $b$ en une unité de temps, de $b^{\prime}$ en $B^{\prime}$ en 0,50 unité, et 
de $b^{\prime \prime}$ en $B$ en 2 unités. La méthode graphique indiquée par M. BERGERON pour l'étude du problème est la suivante:

\section{Remarque préliminaire.}

$1^{\circ}$ L'onde $F$ issue de $A$ à un instant $p-1$ arrive en $b$ à l'instant $p$ : le point $p_{b}$ est donc situé sur la droite $\Phi$ issue du point $(p-1)_{A}$

$2^{\circ}$ L'onde $F^{\prime}$ partie de $b^{\prime}$ à l'instant $p-1$ arrive en $\mathrm{B}^{\prime}$ à l'instant $\mathrm{P}-1+0,5$ et la surpression étant constamment nulle en $B^{\prime}$, le point $(p-1+0,5)_{B}$, est donc situé à l'intersection de l'axe des abscisses, et de la droite $\Phi$ ' issue du point $(p-1)_{t}$. De même, l'onde $f^{\prime}$ partie de $B^{\prime}$ à l'instant $p-1+0,5$ arrive en $D^{\prime}$ à l'instant $p$ et le point de fonctionnement $p_{b}$ ' est donc situé sur la droite $\omega^{\prime}$ issue du point $(p-1+0,5)_{B^{\prime}}$. Autrement dit, le point $p_{b^{\prime}}$ est situé sur la droite $\varphi^{\prime}$ que donne, par réflexion sur l'axe des abscisses, la droite $\Phi^{\prime \prime}$ issue du point $(p-1)_{b^{\prime}}$.

$3^{\circ}$ En raisonnant de la même façon, on verrait que le point $P_{b \text { " }}$ est situé sur la droite "que donne, par réflexion sur l'axe des abscisses, la droite $\Phi^{\prime \prime}$ issue du point $(p-4) b$ "

$4^{\circ}$ Les points $F_{b}, p_{b^{\prime}}, F_{b^{\prime \prime}}$, doivent avoir même ordonnée, la surpression étant la même, à chaque instant, en $b, b^{\prime}, b^{\prime \prime}$. Par contre, les abscisses q, $\mathrm{q}^{\prime}, \mathrm{q}^{\prime \prime}$ de ces trois points sont différentes, et doivent satisfaire à la relation : $q=q^{\prime}+q^{\prime \prime}$.

$5^{\circ}$ Si les points $(p-1)_{A},(p-1)_{b^{\prime}},(p-4)_{b^{\prime \prime}}$ sont connus, les remarques précédentes permettent de déterminer facilement $p_{b}, p_{b^{\prime}}, p_{b^{\prime \prime}}$. Chacun de ces points étant situé sur une droite inclinée $\Delta, \Delta^{\prime}$ ou $\Delta^{\prime \prime}$ bien déterminée, il suffit de déplacer normalement à l'axe des débits une règle parallèle à cet axe jusqu'au moment où les points d'intersection de cette règle avec les trois droites $\triangle, \Delta^{\prime}, \Delta^{\prime \prime}$ auront des abscisses $q, q^{\prime}, q^{\prime \prime}$, telles que l'on ait $q=q^{\prime}+q^{\prime \prime}$ : les positions de $P_{b}, P_{b^{\prime}}, P_{b^{\prime \prime}}$ se trouvent alors déterminées.

$6^{\circ}$ L'onde $f$ partie de $b$ à l'instant $(p-2)$ arrive en $A$ à l'instant $(p-1)$ : le point $(p-1)_{A}$ est donc à l'intersection de la droite o issue du point $(p-2)_{b}$ et de la parabole $\psi_{p}$ _ caractérisant le degré d'ouverture du distributeur à l'instant $p-1$ (ou de l'axe des surpressions, si, à cet instant, la fermeture est complète).

On voit donc, en rapprochant ce résultat des précédents, que si l'on connaît les points ( $p-2)_{0}$ $(p-1)_{b^{\prime}},(p-4)_{b^{\prime \prime}}$, on en déduit immédiatement les points $p_{b}, P_{b^{\prime}}, p_{b^{\prime \prime}}$, la connaissance de ces points entrainant celle des points de fonc- tionnement correspondants en $\mathrm{A},(\mathrm{P}+1)_{\mathrm{A}}$ par exemple, étant sur la droite o issue de $\mathrm{p}_{\mathrm{b}}$ et sur $\psi_{p}+1$ Autrement dit, si l'on connaît les points de fonctionnement en $b, b^{\prime}, b^{\prime \prime}$, à l'instant $p-1$ et aux instants antérieurs à $p-1$ on en déduit immédiatement les points de fonctionnement en $b, b^{\prime}, b^{\prime \prime}$ et $A$ à l'instant $p$, de telle sorte que la construction peut se poursuivre de proche en proche.

\section{Construction graphique des courbes $(q, \xi)$.}

II n'y a pas d'onde de retour $f$ en $A$ avant l'instant 2 ; donc les points $1_{A}$ et $2_{A}$ sont situés à l'intersection de la droite issue de $\mathrm{O}_{A}(q=50$, $\xi=0$ ) et des paraboles $\psi_{1}$ et $\psi_{2}$.

Les points $2_{b^{\prime \prime}}, 3_{b^{\prime \prime}}, 4_{b^{\prime \prime}}$, et $5_{b^{\prime \prime}}$ sont situés sur la droite $c^{\prime \prime}$ issue de $1_{b \prime \prime}$, lui-même confondu avec $\mathrm{O}_{b^{\prime \prime}}$ ou $\mathrm{O}_{\mathrm{A}}(\mathrm{q}=50, \xi=0)$; puisque l'onde de retour $f^{\prime \prime}$ est nulle en $b^{\prime \prime}$ jusqu'à l'instant 5 .

Le point $2_{b}$, est situé sur la droite $\omega^{\prime}$ issue $d u$ point $\mathrm{O}_{\mathrm{b}^{\prime}}(\mathrm{q}=\mathrm{O}, \xi=0)$ puisque l'onde de retour $f^{\prime}$ est nulle en $b^{\prime}$ jusqu'à l'instant 2 .

Les trois points $2_{b}$ sur la droite $\Phi$ issue de $l_{A}$. $2_{b^{\prime}}$ sur $\varphi^{\prime}$ issue de $O_{b^{\prime \prime}}$, et $2_{b^{\prime \prime}}$ sur $\varphi^{\prime \prime}$ issue de $O_{A}$, ayant même ordonnée et des abscisses telles que $q=q^{\prime}+q^{\prime \prime}$ se déterminent immédiatement par le procédé de la règle, indiqué dans la remarque préliminaire.

Les trois points $3_{\mathrm{b}}$ sur la droite $\Phi$ issue de $2_{\mathrm{A}}$.

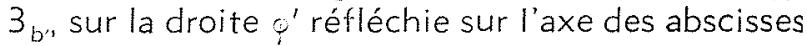
par la droite $\Phi^{\prime}$ issue de $2_{b^{\prime}}$, et $3_{b^{\prime \prime}}$ sur la droite $\phi^{\prime \prime}$ issue de $O_{A}$, se déterminent immédiatement par le même procédé

De $2_{b}$, on déduit $3_{A}$ sur la parabole $\psi_{3}$ et la droite $\hat{o}$ issue de $2_{\mathrm{b}}$

Les trois points $4_{b}$, sur la droite $\Phi$ issue de $3_{A}, 4_{b}$, sur la droite $\varphi^{\prime}$ réfléchie sur l'axe des abscisses par la droite $\Phi^{\prime}$ issue de $3_{b^{\prime}}$ et $4_{b^{\prime \prime}}$ sur la droite ${ }^{\prime \prime}$ issue de $O_{A}$, se déterminent comme précédemment.

Nous avons vu, dans la remarque préliminaire, que l'on détermine facilement $p_{b}, P_{b^{\prime}}, P_{b^{\prime \prime}}$.à partir des points $(p-2)_{b}(p-1)_{b^{\prime}} \cdot(p-4)_{b^{\prime \prime}}$ Cette détermination peut donc jouer à partir de $p=5$, puisque nous connaissons les points $3_{b}, 4_{b^{\prime}}, l_{b^{\prime \prime}}$.

La construction graphique, poursuivie de proche en proche, est reproduite sur la figure 3 , où elle correspond à une mancuvre de fermeture linéaire de durée 7 secondes, soit :

$$
20 \times \frac{0,70}{2}=20 \times \frac{9}{2}
$$



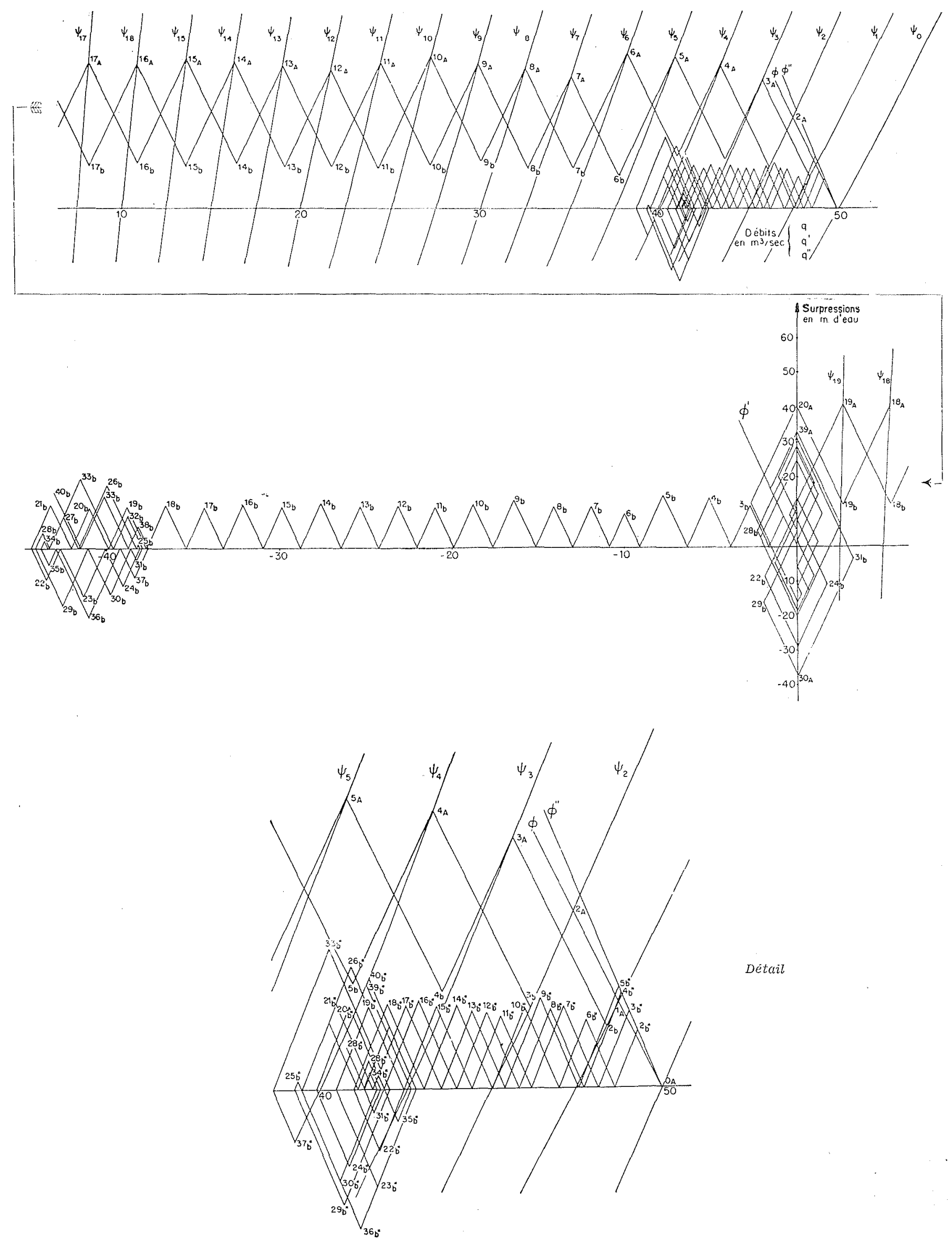

fig. 3 (ef. page suivante) 


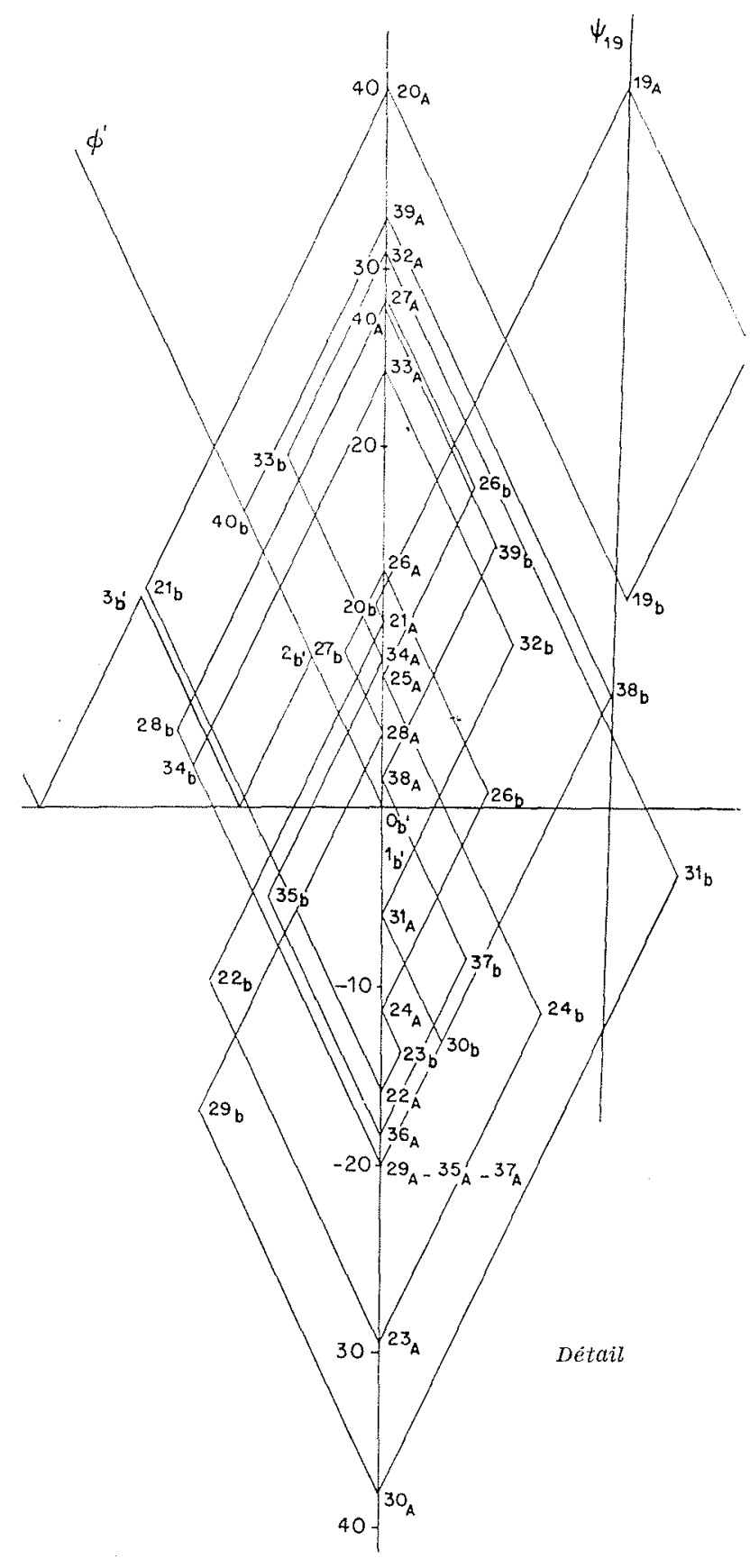

fig. 3

ou 20 intervalles de temps égaux à l'unité adoptée pour la construction graphique.

\section{Résultâts.}

La figure 4 donne les courbes de surpression en $A$ et en $b$, en fonction du temps, ainsi que les débits $q, q^{\prime}, q^{\prime \prime}$ en $b, b^{\prime}, b^{\prime \prime}$. On voit que la surpression due au coup de bélier d'onde, pour cette fermeture linéaire en 7 secondes, varie, en mètres d'eau :

de $-37,5 \mathrm{~m}$. ̀े $+43,5 \mathrm{~m}$. en $A$

de $-20 \mathrm{~m}$. ̀̀ $+20 \mathrm{~m}$. en $b, b^{\prime}, b^{\prime \prime}$,

la hauteur de chute adoptée dans les calculs étant :

$$
1.778-1.558: 220 \mathrm{~m} \text {. }
$$

Les mêmes calculs ont été effectués en supposant la durée de fermeture égale à 4,9 secondes seulement: les figures 5 et 6 montrent les résultats obtenus dans ce cas, pour lequel la surpression en mètres d'eau varie de :

$$
\begin{aligned}
& \text { - } 50 \mathrm{~m} \text {. à }+64 \mathrm{~m} \text {. en } A \\
& \text { - } 21,5 \mathrm{~m} \text {. ̀̀ }+23,5 \mathrm{~m} \text {. en } b, b^{\prime} b^{\prime \prime} .
\end{aligned}
$$

\section{Remarque.}

II serait facile de tenir compte des pertes de charge en associant à chaque droite $\varphi, \varphi^{\prime}, \varphi^{\prime \prime}$ la parabole déformée des pertes de charge correspondantes suivant le procédé classique de M. BERGERON.

De même, il serait facile de tenir compte de la variation du plan d'eau dans la chambre d'équilibre, puisque l'abscisse du point $p_{b}$ donne le débit qui pénètre dans cette chambre, à l'ins$\tan t_{p}$

\section{CHAPITRE IV}

\section{CALCUL DES SURPRESSIONS TRANSMISES AU CANAL D'AMENÉE PAR UNE CHAMBRE D'ÉQUILIBRE DE TRES GRANDE SECTION MUNIE D'UN ÉTRANGLEMENT INFÉRIEUR}

A

La méthode de calcul de $M$. BERGERON exposée au chapitre précédent à propos de l'usine de Brévières, est immédiatement applicable au calcul des surpressions transmises au canal d'amenée par une chambre d'équilibre de section quasi infinie, munie à sa base d'un étranglement inférieur $\mathrm{C}^{\prime}$.

La longueur L' étant ici très faible, $O^{\prime}$ devient très petit par rapport à $\theta$ et $\theta$ ": par exemple, avec les données des (1) Brévières, si l'on rem-

\footnotetext{
(1) La longueur de $1,16 \mathrm{~m}$. adoptée pour l'étranglement peut paraitre forte, surtout si l'on songe aux dispositifs constitués par un simple orifice découpé dans une tôle de 2 a $3 \mathrm{~cm}$. d'épaisseur. II convient toutefois de noter qu'au débouché d'un tel orifice les ondes de surpression cessent d'être planes, ce qui conduit à introduire dans le calcul une longueur fictive de l'étranglement nettement supérieure à sa longueur réelle.
} 


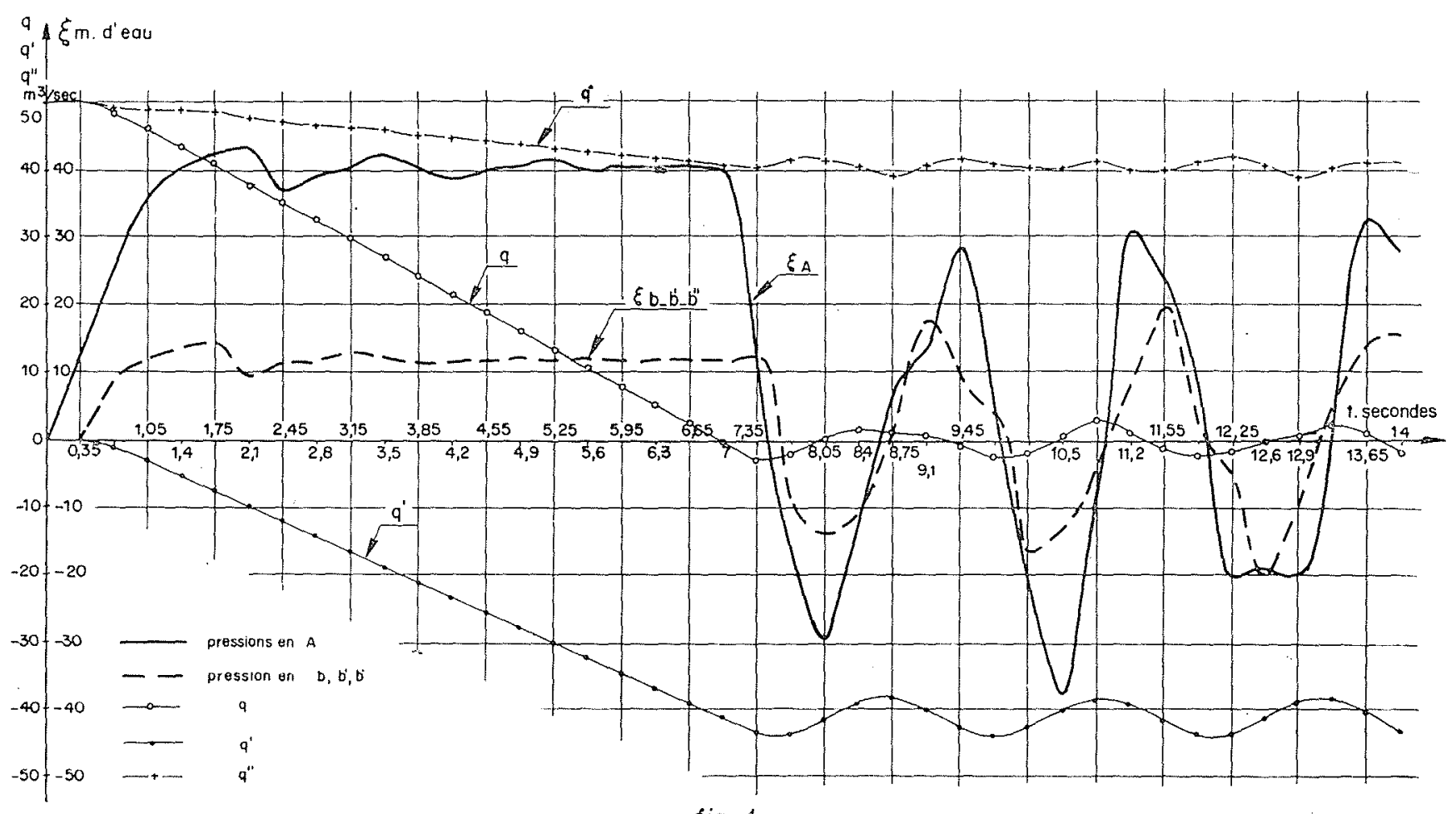

fig. 4
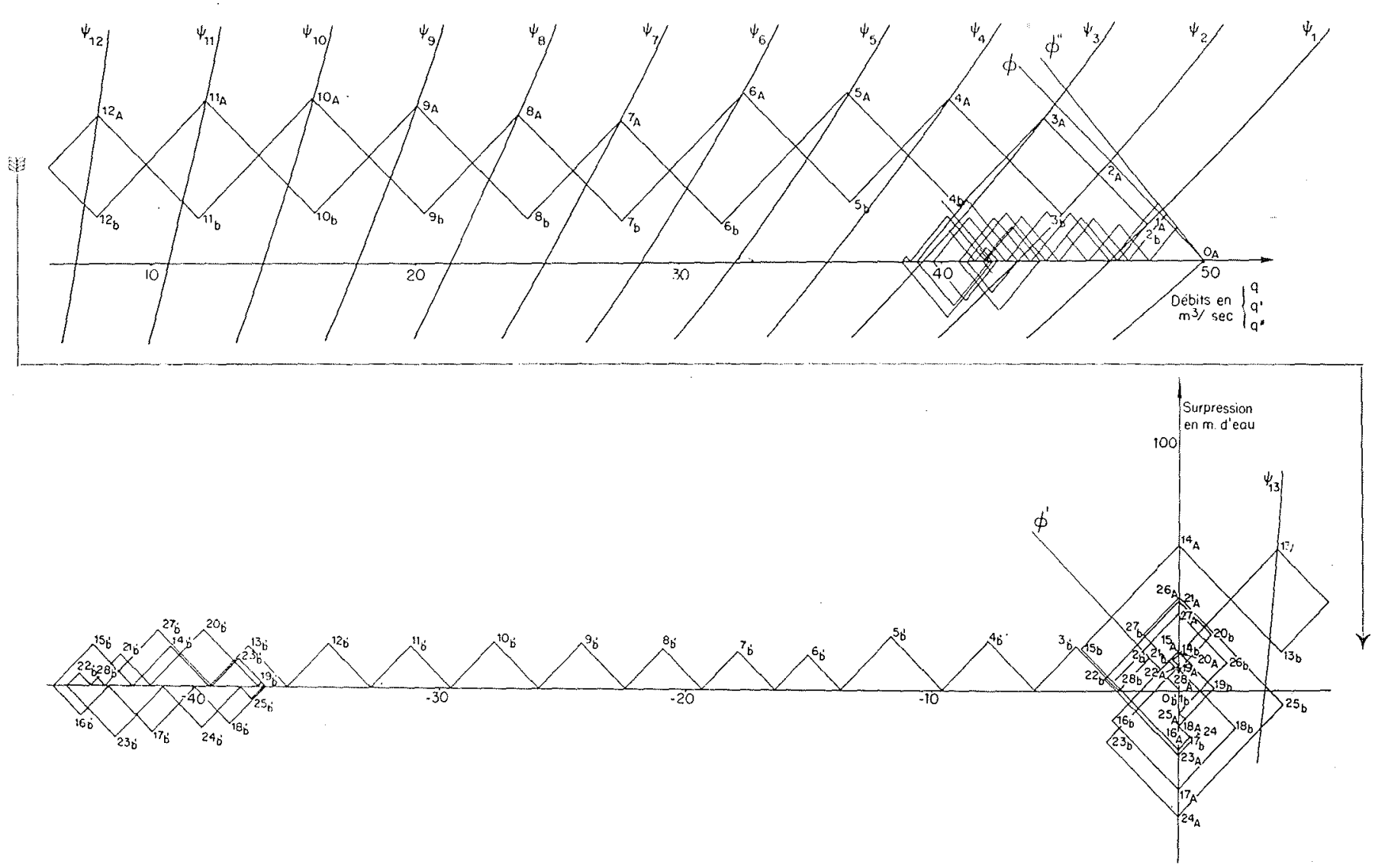

fig. 5 (cf. page suivante) 


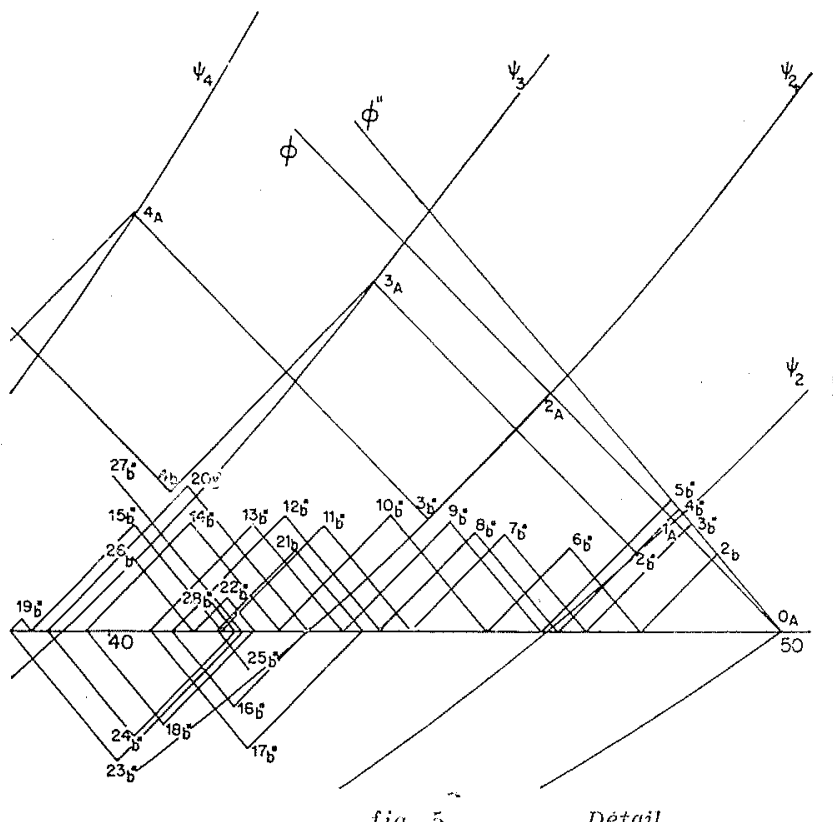

fig. : $\quad$ Détail plaçait la conduite $C^{\prime}$ par un étranglement tel que $L^{\prime}=1,16 \mathrm{~m}$, $a^{\prime}=1.320 \mathrm{~m} . S^{\prime}=3,23 \mathrm{~m}^{2}$, on aurait:

$$
\sigma^{\prime}=1,75 \times 10^{-3}=\frac{0}{400}=\frac{6^{\prime \prime}}{800}
$$

II suffirait de refaire la construction graphique des figures 4 et 6 avec ces nouvelles données, en prenant comme unité de temps la nouvelle valeur de $g^{\prime}$, pour se rendre compte que la surpression obtenue avec une fermeture linéaire en 4,9 secondes, par exemple, serait pratiquement nulle. Nous pouvons nous dispenser d'effectuer cette vérification directe en nous appuyant sur les résultats qui seront exposés dans la suite (voir p. 468).

Nous pouvons donc conclure que, pour toutes les manouvres de fermeture lente, la présence de l'étranglement ne provoque la transmission

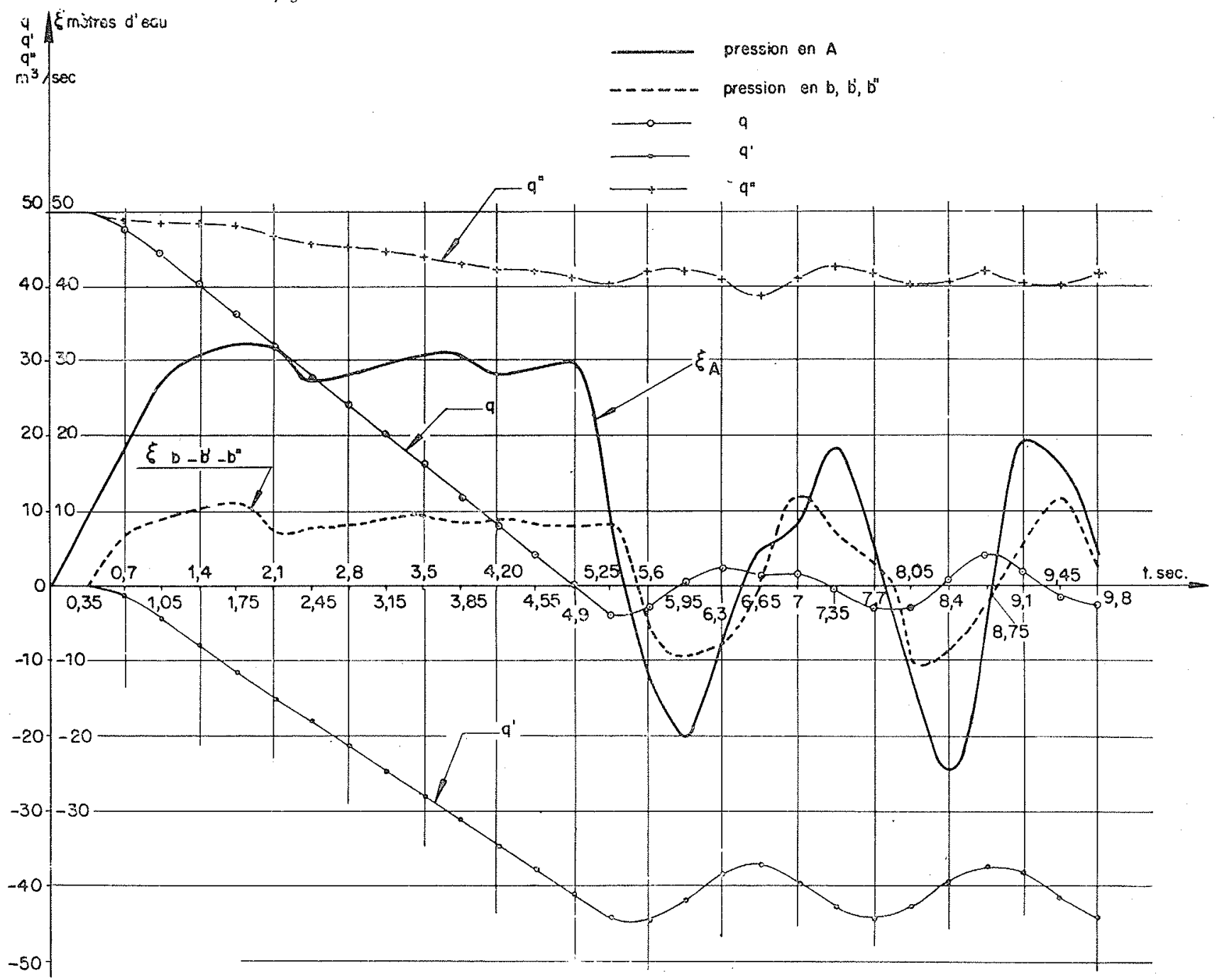

fig. 6 
d'aucune onde de surpression appréciable au canal d'amenée.

\section{B}

Considérons maintenant le cas de fermeture accidentelle de très courte durée en nous placant, pour simplifier, dans les conditions suivantes:

- La fermeture est linéaire en fonction du temps et d'une durée totale ng' très courte, et très inférieure, en particulier, à la durée d'un aller retour d'onde le long de la conduite.

- Le canal d'amenée est supposé nettement plus long que la conduite forcée, de telle sorte que nous ne tenons pas compte, dans l'étude des premières phases du phénomène, des réflexions de l'onde en tête du canal d'amenée: autrement dit, nous effectuons les calculs comme si $\theta^{\prime \prime}$ était infini.
- Nous admettons, en premier lieu, que la caractéristique du canal d'amenée est égale à celle de la conduite forcée $\beta^{\prime \prime}=\beta$.

- Dans une première approximation, nous négligeons la perte de charge à la traversée de l'étranglement.

- Nous nous limitons, tout d'abord, à l'étude de la première onde transmise au canal d'amenée.

- Nous négligeons la variation de pression due au déplacement du niveau dans la chambre d'équilibre.

- La section de la chambre d'équilibre est supposée quasi infinie, de telle sorte que la surpression est constamment nulle audessus de l'étranglement.

Les données du problème sont, par hypothèse, les suivantes:

$$
\begin{aligned}
& y_{1}=220 \mathrm{~m} ., \quad q_{0}=50 \mathrm{~m}^{3} / \mathrm{sec} . \\
& a=1.000 \mathrm{~m} . / \mathrm{s} ., \quad S=9,82 \mathrm{~m}^{2}, \quad \frac{\mathrm{a}}{\mathrm{gS}}=10,40 . \\
& a^{\prime}=1.320 \mathrm{~m} . / \mathrm{s} ., \quad S^{\prime}=3,23 \mathrm{~m}^{2}, \quad \frac{\mathrm{a}^{\prime}}{\mathrm{gS}^{\prime}}=41,60 . \\
& \mathrm{L}^{\prime}=1,16 \mathrm{~m} ., \quad \theta^{\prime}=\frac{2 \mathrm{~L}^{\prime}}{\mathrm{a}^{\prime}}=1,75 \times 10^{-3} \mathrm{~s} .
\end{aligned}
$$

L'hypothèse $\xi^{\prime \prime}=\beta$ se traduit par le fait que, sur le diagramme, les droites $"$ et $\varphi$ ont même pente.

II est évidemment anormal de considérer, comme nous le faisons ici pour l'étude de fermetures extrêmement rapides, un régime permanent initial de débit $q_{0}=50 \mathrm{~m}^{3} / \mathrm{sec}$., égal au débit maximum de l'usine. Cette hypothèse, qui conduit à des valeurs considérables de la surpression au distributeur $\left(\frac{\mathrm{aq}_{0}}{\mathrm{gS}}=520 \mathrm{~m}\right.$ ) pour une pression statique $y_{0}=220 \mathrm{~m}$. (2,36 fois moindre), est, fort heureusement, irréalisable en pratique, de telles mancuvres, même accidentelles, ne pouvant intéresser qu'une faible fraction du débit maximum. Cette base de calcul a toutefois l'avantage de nous permettre d'exagérer, pour la rendre plus nettement perceptible, l'influence de certains éléments, tels que la perte de charge dans l'étranglement, qui, pour de faibles débits, est presque inappréciable. Ce mode opératoire n'est d'ailleurs pas gênant, puisque, dans le tableau final des résultats, nous exprimons le maximum de l'onde transmise au canal d'amenée, dans chaque cas considéré, en pour cent de la surpression maximum au distributeur, cette dernière étant elle-même proportionnelle au débit du régime permanent initial correspondant.

La construction graphique reste la même que celle qui a été exposée au chapitre précédent à propos du problème des Brévières; les hypothèses faites au sujet du canal d'amenée introduisent une simplification: l'onde de retour $f^{\prime \prime}$ étant constamment nulle en $b^{\prime \prime}$, les points de fonctionnement en $b^{\prime \prime}$ se trouvent tous sur la droite $\rho^{\prime \prime}$ issue du point initial $O_{A}$, droite $\rho^{\prime \prime}$ qui est elle-même confondue avec la droite issue du même point. 
Certaines remarques facilitent la construction. Tout d'abord, au lieu de tracer les paraboles résultant de la loi linéaire de fermeture, il est plus simple de calculer directement, pour placer les droites nécessaires à la construction, les abscisses des points d'intersection de ces paraboles avec la droite issue du point $O_{A}$. On trouve que l'abscisse du point d'intersection $p_{A}^{\circ}$ correspondant à l'instant $t_{f}$. est (fig. 7).

$$
\begin{aligned}
& q_{p}^{0}=\frac{2\left(y_{0}+q_{0}+g a\right)}{\operatorname{tg} \alpha} \times \frac{1}{1+\sqrt{1+\frac{4 n^{2} y_{0}\left(y_{0}+q_{0}^{\dagger} g \alpha\right)}{\operatorname{tg}^{2} \alpha \cdot q_{0}^{2}} \times \frac{1}{(n-p)^{2}}}} \\
& \text { avec tg } \alpha=\frac{a}{g S}
\end{aligned}
$$

Ensuite, on peut remarquer qu'à partir de l'instant $t_{x}$ postérieur de $\theta / 2$ à celui où la fermeture s'achève au distributeur, le point de fonctionnement $p_{b}($ avec $p>n)$, se déplace sur la droite $\Phi$ issue du point $\left[q=0, \xi=\frac{a v_{0}}{g}\right]$ où la droite $\varphi$ issue de $\mathrm{O}_{\mathrm{A}}$ coupe l'axe $\mathrm{O} \xi$ : dans tous les cas où $T$ est faible vis-à-vis de $\theta$ le point de fonctionnement correspondant $p_{\partial^{\prime \prime}}$, de même ordonnée, étant toujours sur cette droite issue de $\mathrm{C}_{\mathrm{A}}$, on a $\mathrm{q}^{\prime \prime}=-\mathrm{q}$, et la relation $\mathrm{q}=\mathrm{q}^{\prime}+\mathrm{q}^{\prime \prime}$ donne $q^{\prime}=+2 q$, ce qui montre que le lieu $d u$ point $p_{b^{\prime}}($ avec $p>n)$ est la droite passant par les points $\left(\mathrm{q}=0, \xi=\frac{a v_{0}}{\mathrm{~g}}\right)$ et $\left(\mathrm{q}=-2 \mathrm{q}_{0}\right.$ $\xi=0)$

La figure 7 représente la construction relative à une fermeture effectuée en une durée
$T=0,175 \mathrm{sec} .=100 \mathrm{fj}^{\prime}:$ une telle fermeture du débit maximum en moins de 2 dixièmes de seconde, irréalisable en pratique, correspondrait, comme nous l'avons vu, au distributeur, à une énorme surpression de $520 \mathrm{~m}$. supérieure au dou'bie de la pression statique. La surpression maximum sous le diaphragme atteindrait, dans ce cas, $=36,25 \mathrm{~m}$.

Des constructions graphiques analogues ont été effectuées pour des fermetures de plus en plus brèves, de durées respectivement égales à $50 \mathrm{gl}$, $20 g^{\prime}, 10 g$ ' et 0 , engendrant des surpressions croissantes sous l'étranglement comme le montrent les premières lignes du tableau 1.

Sur la figure 8, sont reportées les courbes donnant, en fonction du temps, la surpression sous le diaphragme, dans chacun des cas ainsi étudiés.

C

On peut obtenir facilement une valeur approchée par excès de la surpression $\xi_{M}$, suffisamment précise dès que la durée de fermeture $T$ est supérieure à $50 \mathrm{o}^{\prime}$, soit, ici, à moins d'un dixième de seconde, en faisant la remarque suivante, inspirée par exemple, par l'examen de la figure 7 . La surpression maximum $\zeta_{M}$ est produite dans le $n^{\text {me }}$ intervalle de temps $O^{\prime}$ considéré, c'est-à-dire dans celui qui correspond à la fin de la fermeture: la courbe ( $q, \ldots$ ) est alors presque horizontale et la distance horizontale des points $(n-1)_{b} r_{b}$, égale par ailleurs à $\frac{2 \xi_{M}}{\operatorname{tg}_{x}{ }^{\prime}}$, est sensiblement la même que la

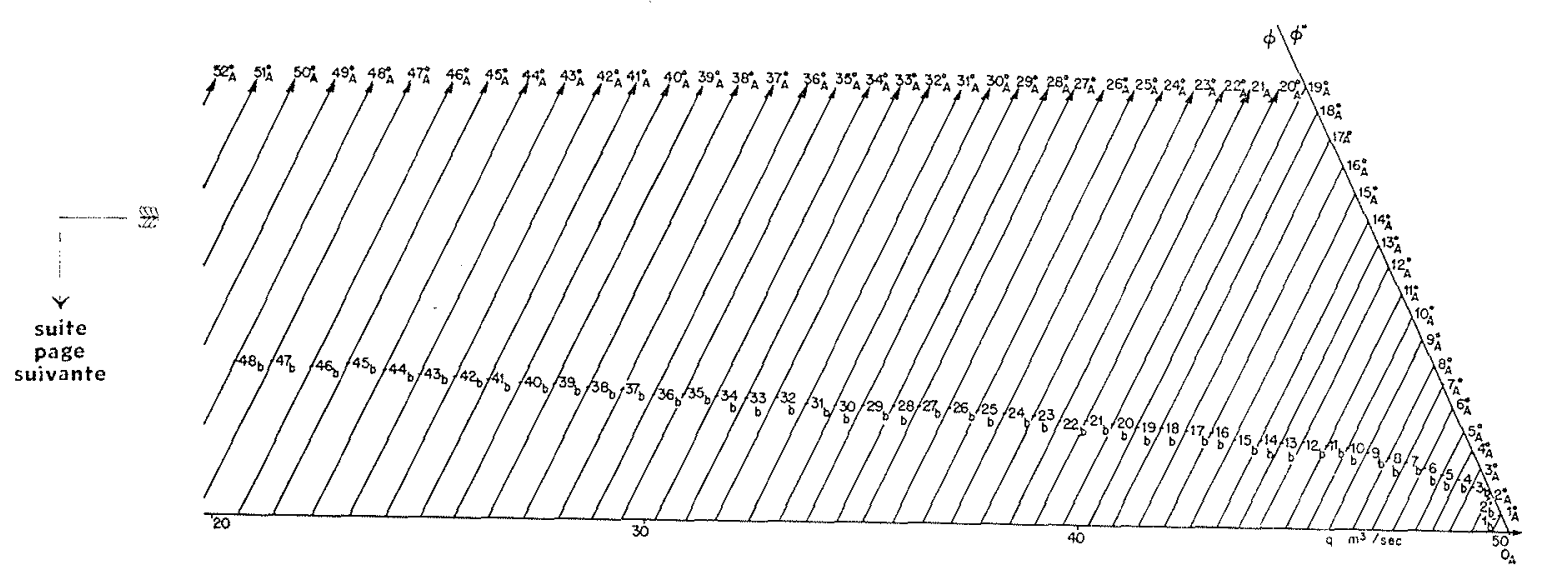

fig. 7 

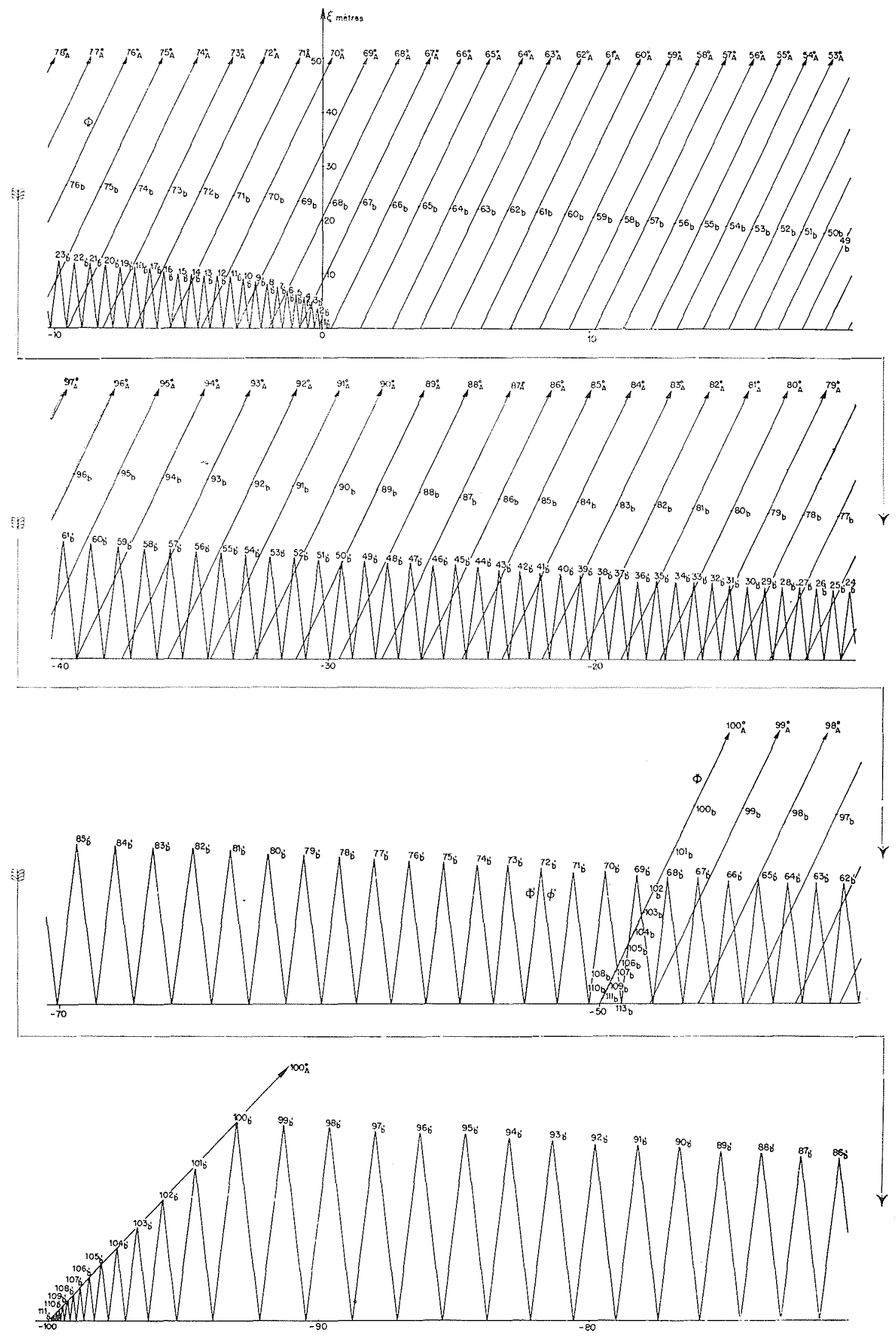

fig. 7 


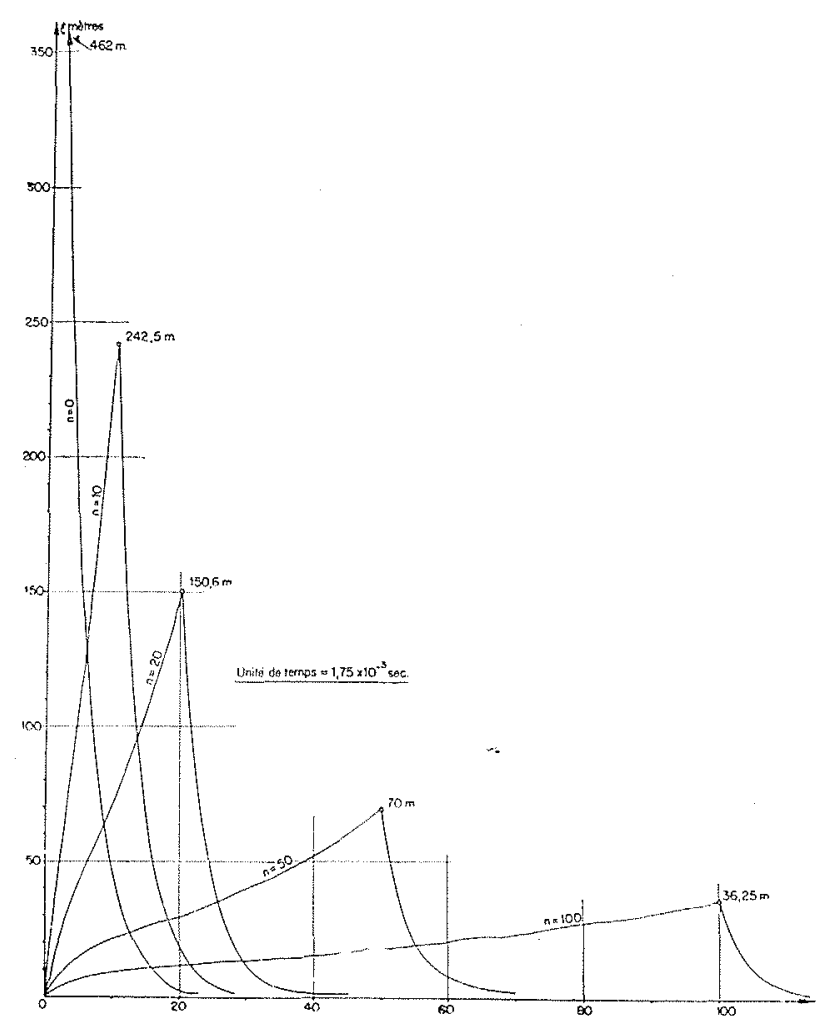

fig. 8

distance horizontale des droites $\Phi$, passant par les point $(n-1)_{A}^{\circ}$ et $n_{A}^{\circ}$, distance qui représente elle-même le double de l'abscisse $q_{n}^{0} \ldots$ i. du point $(n-1)_{A}^{\circ}$, donnée par l'expression (6) en faisant dans celle-ci $p=n-1$. On tire de là l'expression suivante de $\zeta_{M}$ :

$$
\begin{aligned}
& \xi_{M}=\frac{\operatorname{tg} \alpha^{\prime}}{\operatorname{tg} \alpha} \times \frac{2\left(y_{0}+q_{0} \lg \alpha\right)}{1+\sqrt{1+\frac{4 y_{0}\left(y_{0}+q_{0} \operatorname{tg} \alpha\right) n^{2}}{q_{0}^{2} \operatorname{tg}^{2} \alpha}}} \\
& \text { avec: } \operatorname{tg} \alpha^{\prime}=\frac{a^{\prime}}{g s^{\prime}}, \operatorname{tg} \alpha=\frac{a}{g s}
\end{aligned}
$$

Sur la figure 9 , nous avons tracé les courbes donnant, en fonction de $n$, pour $O \leqslant n \leqslant 100$, les valeurs de la surpression maximum $\zeta_{M}$ trans. mise au canal d'amenée et celles du rapport $K=\xi_{M} / \frac{a q_{0}}{g S}$ à la surpression maximum $\frac{a q_{0}}{g S}$ au distributeur, les valeurs de $\zeta_{M}$ résultant soit de la construction graphique exacte, soit de la formule approchée (7), pour l'exemple ci-dessus étudié: on voit que les

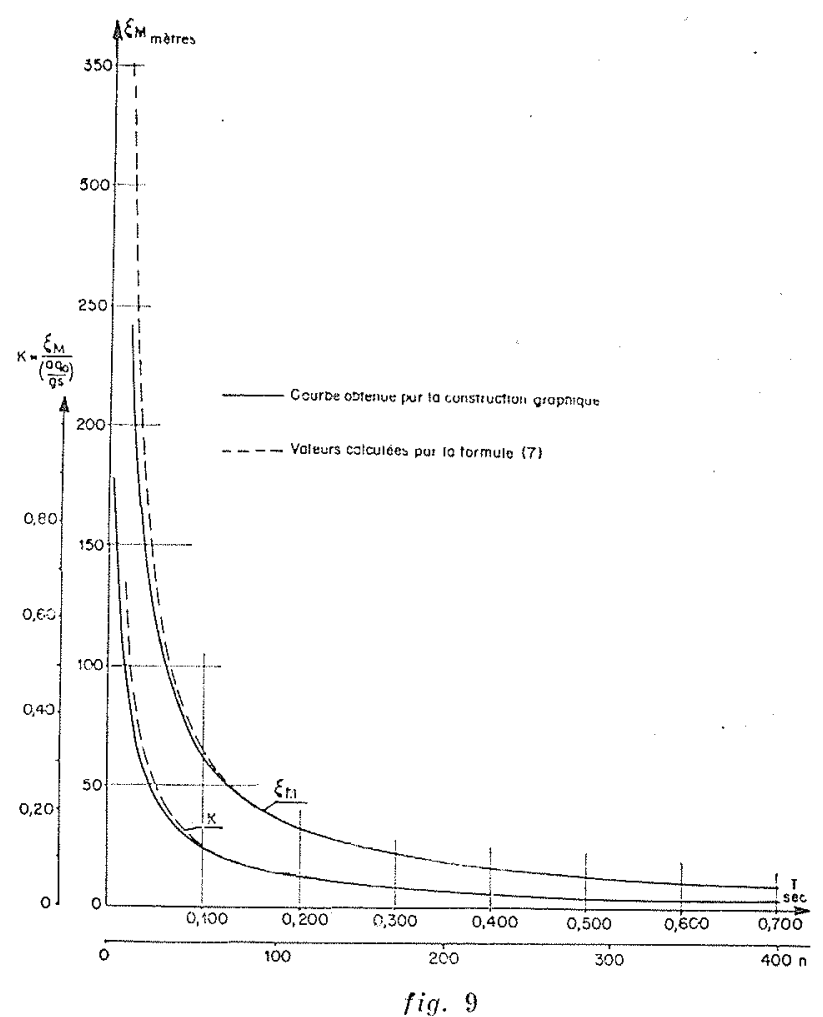

deux courbes sont pratiquement confondues au delà de $n=100$, et que l'erreur, encore très faible pour $n \geqslant 50$, est toujours dans le sens de la sécurité. Les dernières lignes du tableau I (i) explicitent, pour les valeurs élevées de $n$, les valeurs de $\zeta_{M}$ et de $\zeta_{M} / \frac{a q_{0}}{g S}$. On remarque que, pour $n=400$, limite supérieure des fermetures brusques, puisque l'on a ici $j=400$ o', la surpression sous le diaphragme est seulement de $9,35 \mathrm{~m}$, représentant à peine $1,8 \%$ de la surpression correspondante de $520 \mathrm{~m}$. obtenue au distributeur.

On comprend que, pour les manceuvres normales de fermeture, netrement plus longues ef produisant une surpression au distributeur 10 ou 20 rois plus raible, la surpression sous le diaphragme soî̌ pratiquement nulle, ell ceci justifie les conclusions énoncées au début de ce chapitre en ce qui concerne les fermerures lentes, seules réalisées en pratique, à partir d'ume fractron notable du débir maximum.

(1) P. 469 
TABLEAU

\begin{tabular}{|c|c|c|c|}
\hline$n$ & T sec. & $\mathrm{m} \mathrm{m}$ & $K=G M \mid \frac{a q_{1}}{a g}$ \\
\hline 0 & 0 & 462 & 0,89 \\
\hline 10 & 0,0175 & 242,5 & 0,467 \\
\hline 20 & 0,035 & 150,6 & 0,29 \\
\hline 50 & 0,0875 & 70 & 0,134 \\
\hline 100 & 0,175 & 36,25 & 0,0697 \\
\hline 200 & 0,35 & 18,70 & 0,036 \\
\hline 300 & 0,525 & 12,50 & 0,024 \\
\hline 400 & 0,70 & 9,35 & 0,018 \\
\hline
\end{tabular}

D

Dans ce qui précède, rous n'avons pas fait intervenir la perte de charge dans l'étranglement. Il est facile d'en tenir compte, en localisant celle-ci à la section terminale supérieure de l'étranglement, les droites o' venant se réfléchir sur la parabo!e des pertes de charge au lieu de rebondir sur l'axe des débits.

La figure 10 montre la construction obtenue, avec les mêmes données que précédemment, avec $q_{11}=50 \mathrm{~m}^{3} / \mathrm{sec} . \mathrm{n}=10$, et une perte de charge dans l'étranglement $0,006 q^{2}$.

Le maximum de la surpression (fig. 11) atteini maintenant $247.5 \mathrm{~m}$., contre 242,50 quand on néglige la perte de charge. L'influence de la perte de charge est donc, dans ce cas extrêmement faible, presque négligeable.

En faisant la même étude pour une fermeture dix fois plus lente, relative à $n=100$, les autres données restant les mêmes, l'influence de la perte de charge devient très importante comme on le voit sur la figure 12 ; en particulier, le maximum de la surpression passe à $74,8 \mathrm{~m}$., contre $36,25 \mathrm{~m}$. en l'absence de pertes de charge.

L'étude de ce dernier cas pourrait conduire à des conclusions très sévères vis-à-vis de l'étranglement: en effet, l'onde de surpression est accompagnée d'une onde de débit qui, dans le cas actuel, provoque la traversée de l'étranglement par un débit voisin de $2 q_{0}$ et, par suite, superpose à l'onde de surpression proprement dite une surélévation de pression presque quadruple de la perte de charge existant dans l'étranglement pour le débit $q_{r}$. Un tel effet est particulièrement sensible dans le cas actuel parce que nous avons admis que le débit $q_{0}$ intéressé par la fermeture complète est le débit maximum $\mathrm{q}_{0}=50 \mathrm{~m}^{3} / \mathrm{sec}$. absorbé par l'usine. En pratique, des fermetures brusques, au sens que la théorie des coups de bélier attache à ce terme $(T<\theta)$ n'interviennent jamais pour le débit maximum de l'usine: une fermeture en 0,175 secondes, comme celle qui correspond au cas actuel, ne peut intéresser qu'un régime initial correspondant à quelques mètres cubes secondes de débit. Il suffit de remarquer que la perte de charge dans l'étranglement, due à la traversée de celui-ci par un débit sensiblement double de celui qui correspond au régime permanent initial, passe de 60 mètres à 2,4 mètres, lorsque $q_{6}$ passe de $50 \mathrm{~m}^{3} / \mathrm{sec}$. à $10 \mathrm{~m}^{3} / \mathrm{sec}$. pour se rendre compte qu'en pratique l'influence de la perte de charge n'a jamais un caractère menaçant.

\section{$E$}

Revenons au cas où l'on néglige la perte de charge dans l'étranglement. Dans ce qui précède, nous avons considéré seulement la première onde de surpression sous le diaphragme: il est facile de poursuivre la construction pour l'étude des intervalles suivants. C'est ce que montre, pour $\mathrm{q}_{0}=10 \mathrm{~m}^{3} / \mathrm{sec}$ et $\mathrm{n}=10$, en admettant qu'il n'y ait cavitation en aucune section, le graphique de la figure 13, dans laquelle:

$P_{\AA}^{\circ}$ représente le point de fonctionnement en $A$ à l'instant $t_{p}$ fin du pème intervalle de temps of suivant le début de la fermeture.

$p_{b}, p_{b^{\prime}}$ sont relatifs aux sections $b, b^{\prime}$ à l'ins$\tan t t_{p}+\frac{\theta}{2}$.

$P_{A}^{\prime}$ concerne la section $A$ à l'instant $t_{p}+\theta$ $P_{b_{1}}, P_{b_{1}^{\prime}}$, concernent les sections $b, b^{\prime}$ à l'ins$\operatorname{tant} t_{p}+\frac{3 \theta}{2}$.

$P_{A}^{2}$ concerne la section $A$ à l'instant $t_{p}+2 \theta$.

La figure 14 représente les courbes de surpression correspondantes en $A$ et sous l'étranglement, en fonction du temps.

\section{F}

Dans ce qui précède, nous avons supposé, pour simplifier, que l'on avait :

$$
\frac{a^{\prime \prime}}{g S^{\prime \prime}}=\frac{a}{g S}
$$



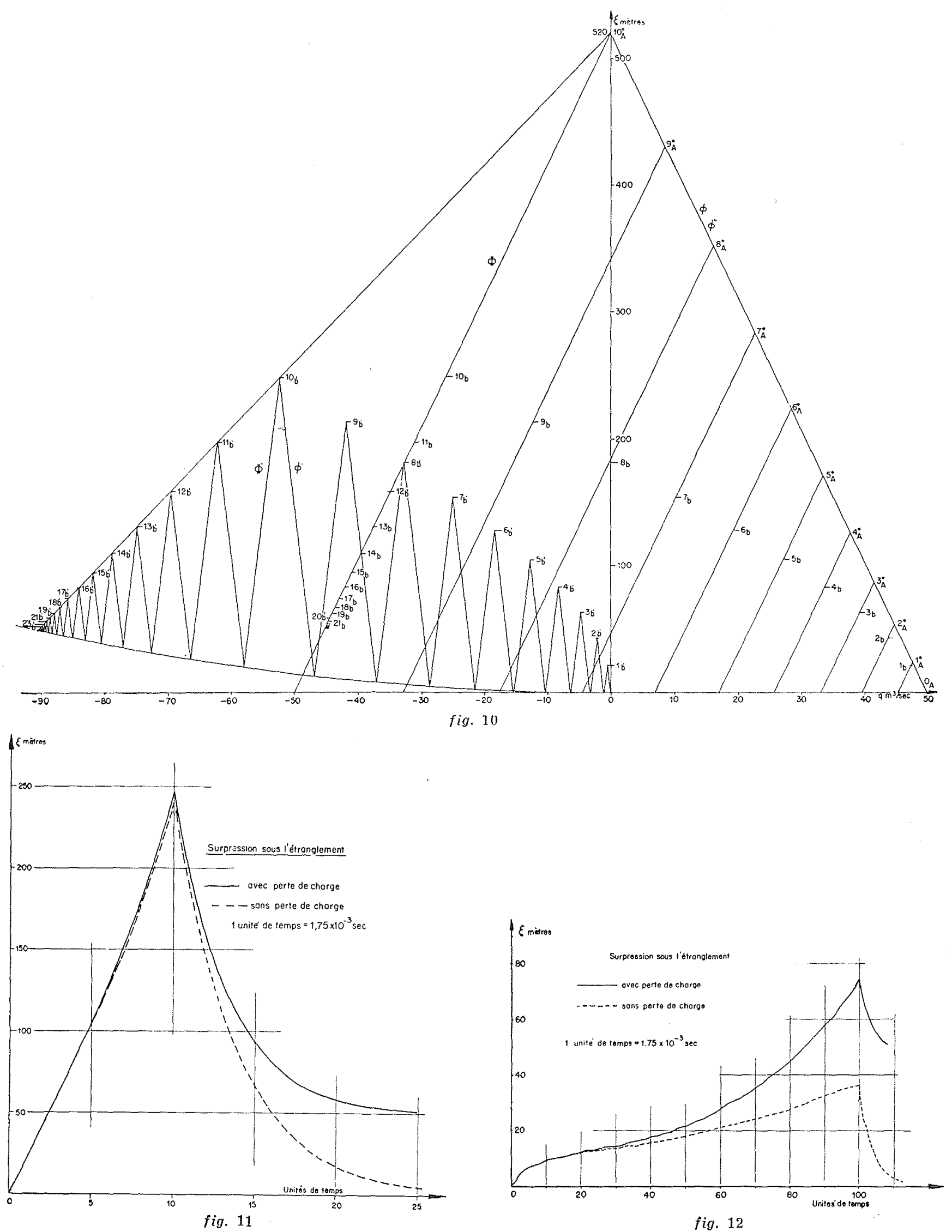

fig. 12 


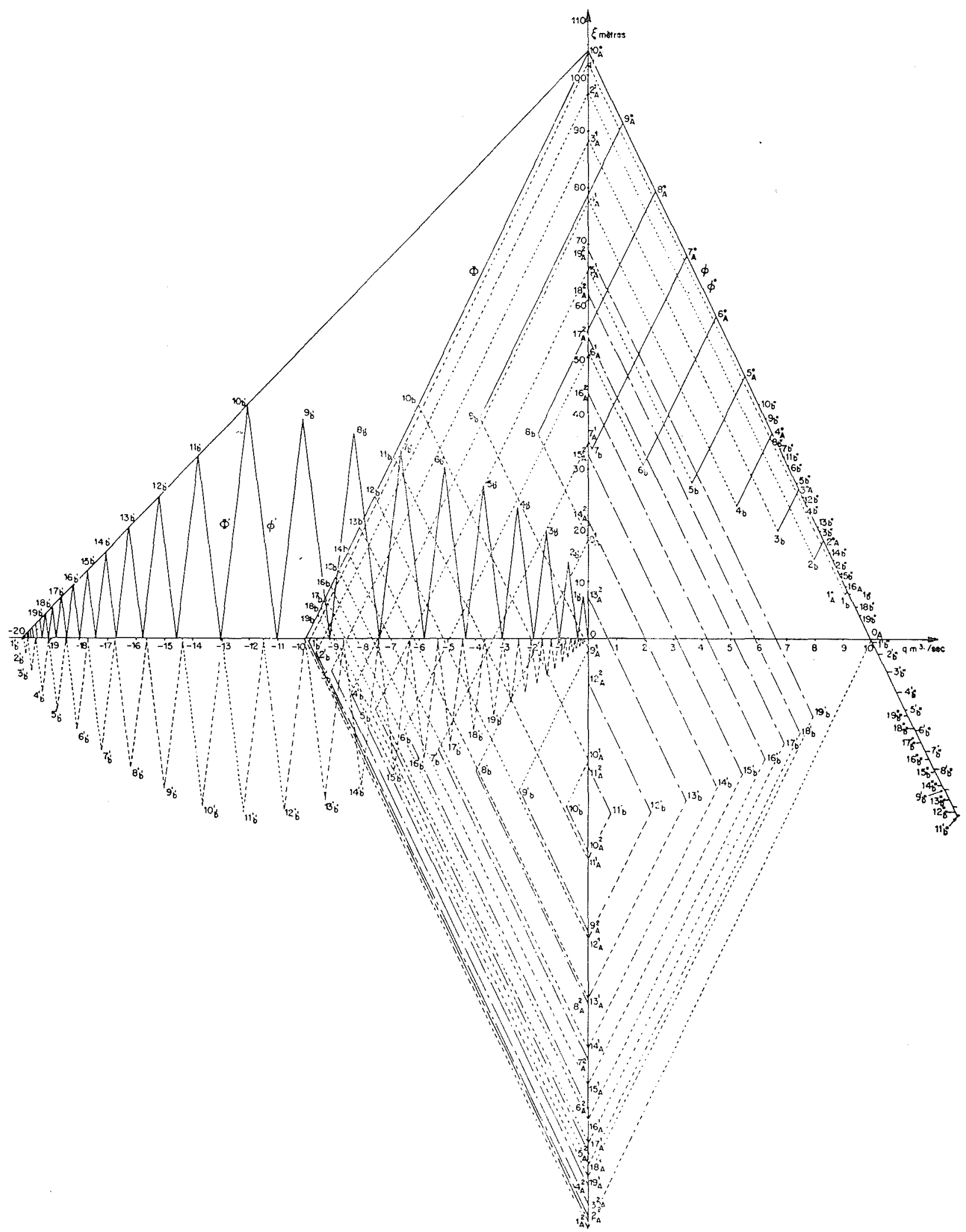

fig. 13 


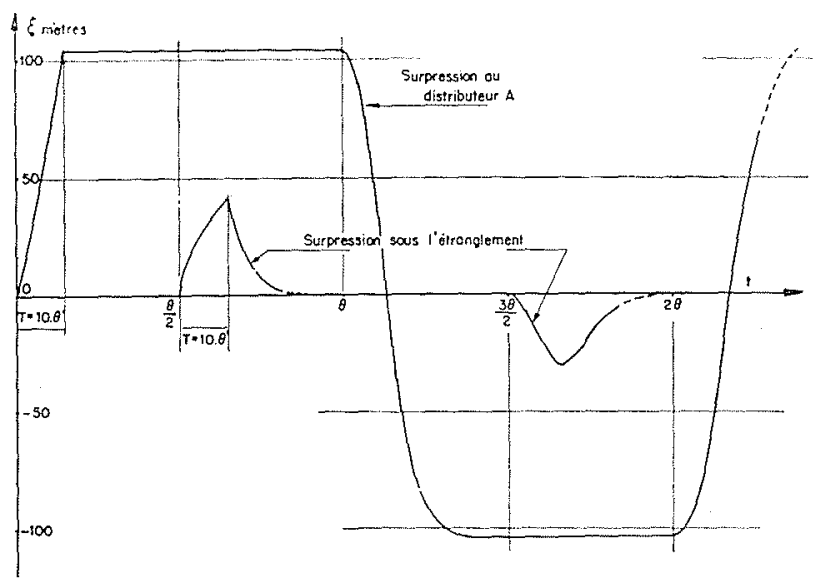

fig. 14
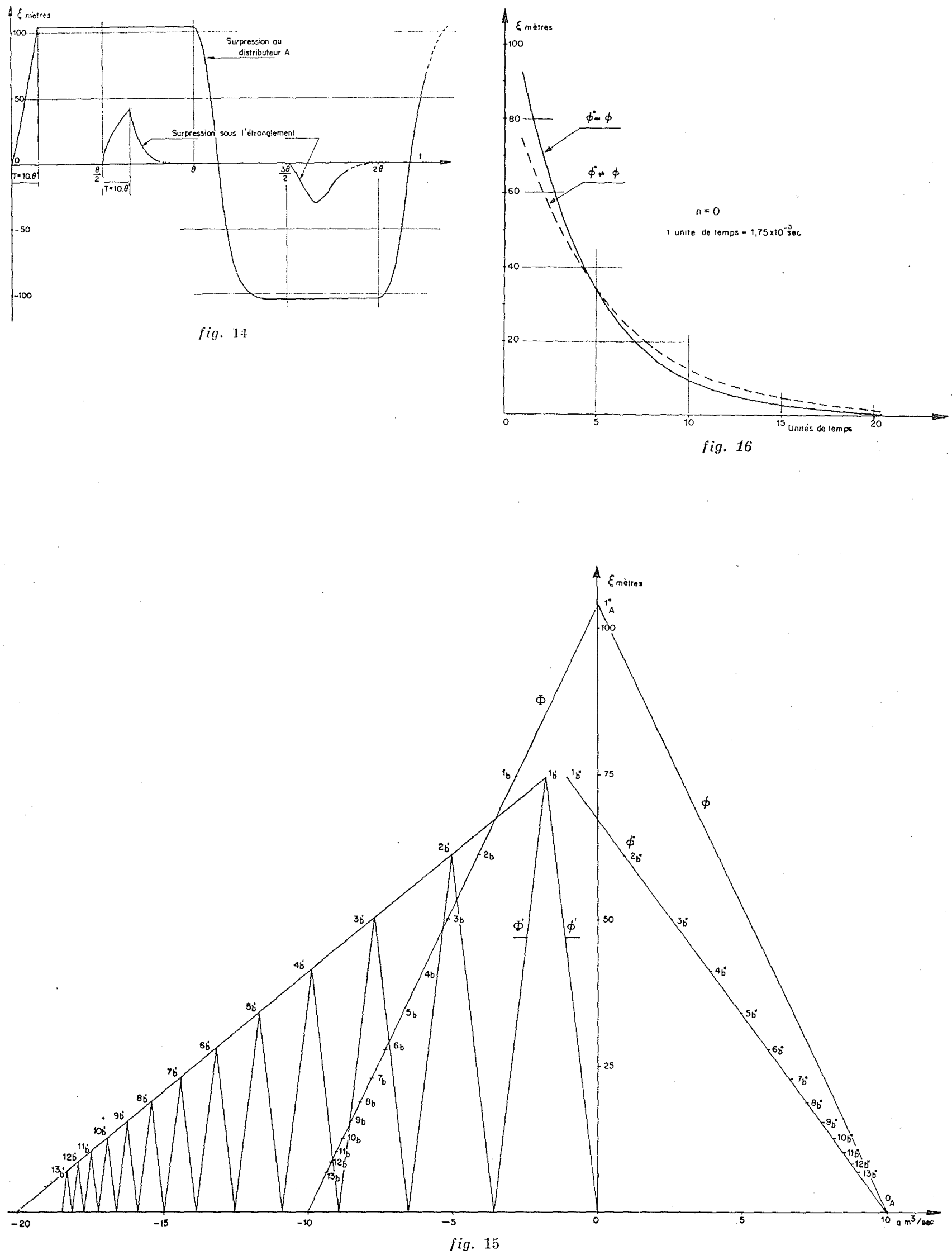


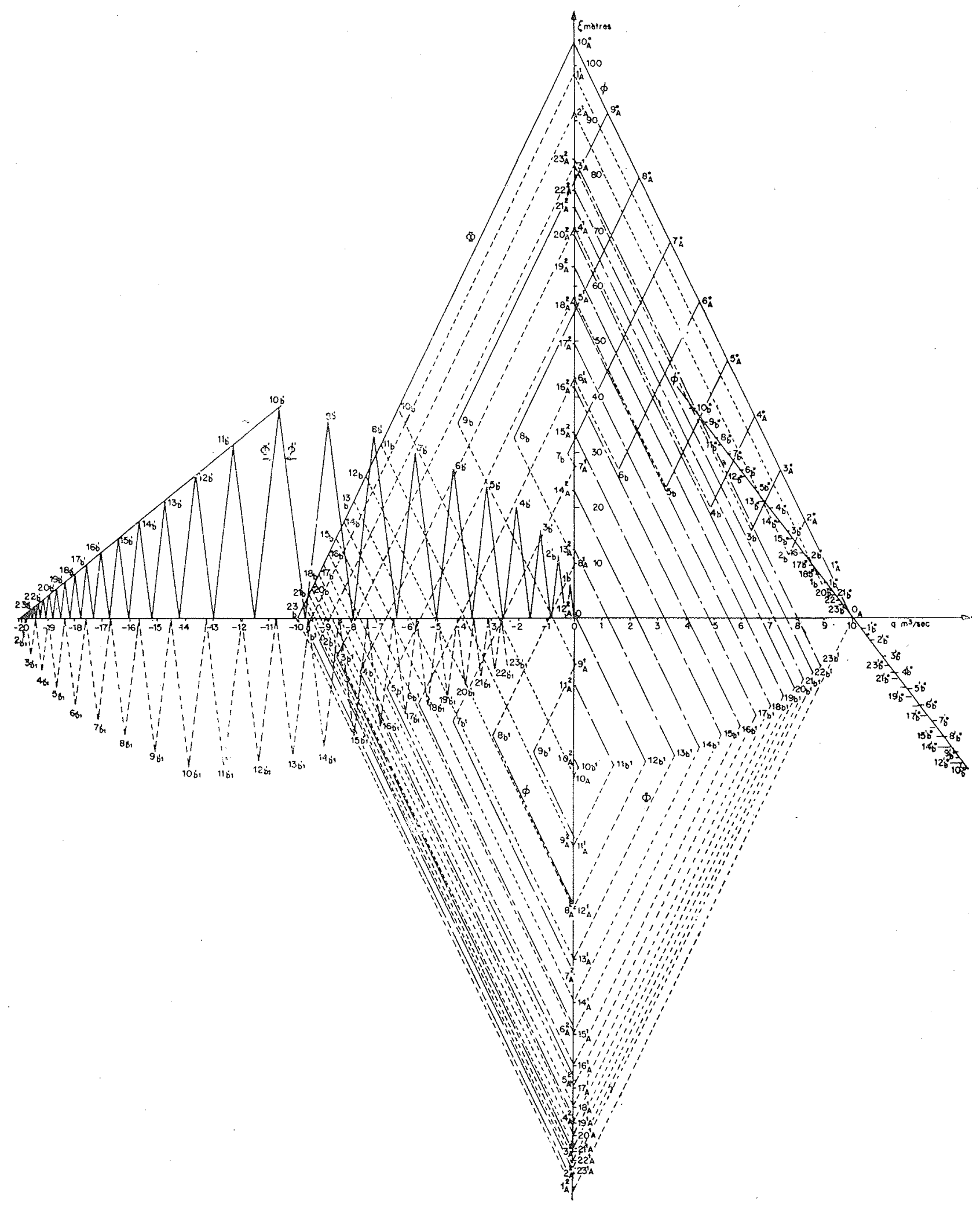

Fig. 17 
Le cas où le canal d'amenée a une caractéristique différente de la conduite forcée s'étudie tout aussi facilement, suivant les mêmes principes: le seul changement vis-à-vis des constructions précédentes, réside dans le fait que le lieu des points de fonctionnement concernant la section $b^{\prime \prime}$ est une droite o passant par $O_{A}$ qui fait un certain angle avec la droite $\phi$ issue de ce même point, au lieu d'être confondue avec elle.

De ce fait, la formule (7) donnant la surpression maximum sous l'étranglement, demeure valable avec le même degré d'approximation que frécédemment.

Les figures 15 et 16 correspondent à la fermeture instantanée du débit de $10 \mathrm{~m}^{3} / \mathrm{sec}$. en négligeant les pertes de charge et en se limitant à la première onde de surpression. Les écarts, visà-vis du cas où les droites et " sont parallèles, sont plus importants que dans l'exemple précédent, $\xi_{M}$ atteignant maintenant $74,5 \mathrm{~m}$. contre $92,4 \mathrm{~m}$. lorsque $\varphi^{\prime \prime}$ et $\varphi$ sont parallèles.

La figure 17 donne la construction relative à $n=10$, en négligeant les pertes de charge, pour l'onde d'aller et l'onde réfléchie au distributeur, avec $q_{0}=10 \mathrm{~m}^{3} / \mathrm{sec}$, et $\frac{\mathrm{a}^{\prime \prime}}{\mathrm{gs}^{\prime \prime}}=6.625$,

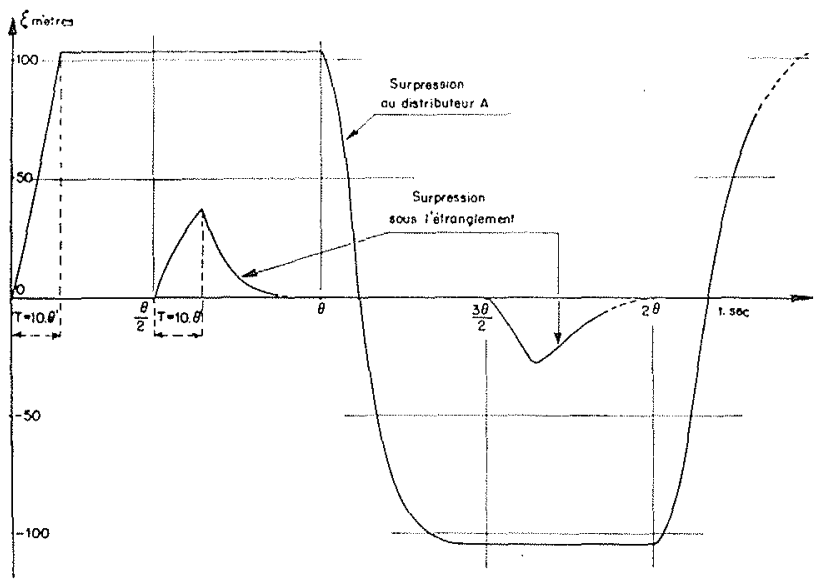

Fig. 18 les autres données demeurant les mêmes que précédemment.

La figure 18 fournit les courbes de surpressions correspondantes : les valeurs extrêmes de sont $+38 \mathrm{~m}$. et $-26,80$ contre $+41,50 \mathrm{~m}$. et - $31,25 \mathrm{~m}$. dans le cas où $\frac{\mathrm{a}^{\prime \prime}}{\mathrm{gS}^{\prime \prime}}$ était égal à $\frac{\mathrm{a}}{\mathrm{gS}}$.

Plus n croît, plus l'écart diminue, le raisonnement fait pour établir l'expression de $\check{\zeta}_{M}$ demeurant entièrement valable dans le cas ou o" n'est pas parallèle à $o$, de telle sorte que la formule (7) donnant $\zeta_{M}$ demeure encore valable pour le cas actuel.

\section{CHAPITRE $V$ \\ CAS OU L'ÉTRANGLEMENT EST ACCOMPAGNÉ \\ D'UNE CONDUITE DE LIAISON DE SECTION LIMITÉE}

\section{A}

Nous avons supposé jusqu'à présent l'étranglement placé directement sur le canal d'amenée et suivi immédiatement d'une chambre d'équilibre de section pratiquement infinie. Le plus souvent, il n'en est pas ainsi et l'étranglement se trouve suivi ou précédé d'une conduite de liaison de quelques mètres de longueur, dont l'effet se combine à celui de l'étranglement pour accroître la surpression transmise au canal d'amenée, dans le cas de fermeture accidentelle de très faible durée. La méthode graphique de M. BERGERON permet de résoudre aisément le problème suivant les mêmes principes que précédemment.

Pour fixer les idées, supposons l'étranglement surmonté du tube de liaison, la chambre d'équilibre ayant elle-même une section assez grande pour que la surpression demeure pratiquement nulle à sa base et retenons les données suivantes:

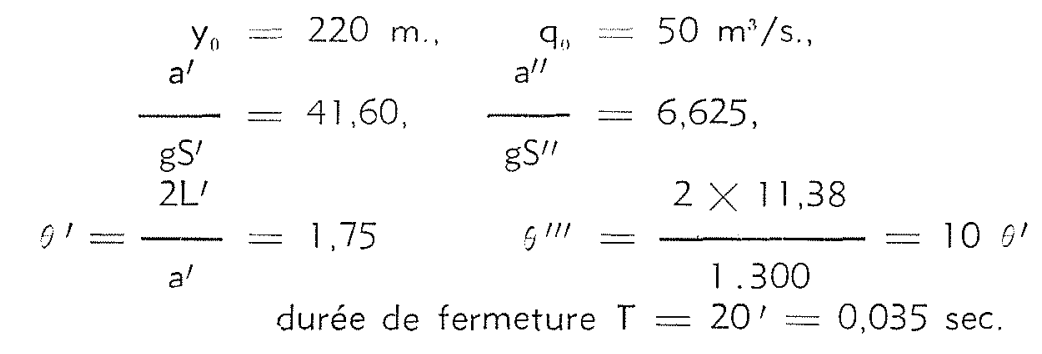

$$
\begin{gathered}
\frac{\mathrm{a}}{\mathrm{gS}}=10,40 \\
\frac{\mathrm{a}^{\prime \prime \prime}}{\mathrm{gS}^{\prime \prime \prime}}(\text { tube de liaison }=6,625)
\end{gathered}
$$




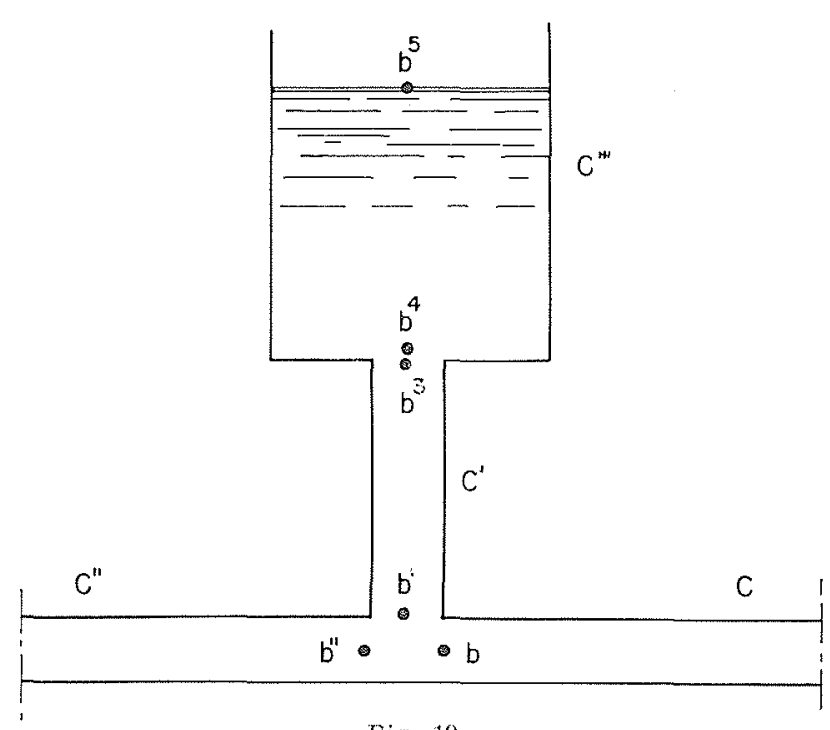

Fig. 19
Le tube de liaison, dans cet exemple, a la même section que le canal d'amenée et sa longueur $L^{\prime \prime \prime}=11,38 \mathrm{~m}$. est parcourue par l'onde en un temps dix fois supérieur à celui que nécessite la traversée de l'étranglement.

Les autres notations sont précisées sur la figure 19.

La construction graphique de la figure 20 ne tient pas compte de la perte de charge dans l'étranglement.

L'onde $F^{\prime}$ partie de $b^{\prime}$ à l'instant $1\left(T_{b^{\prime}}\right)$ arrive en $b^{3}$ à l'instant 1,5:1,5 $b^{\prime}$, confondu avec 1,5 $b^{*}$ (même débit et même surpression) doit être sur la droite $\psi^{\prime \prime \prime}$ issue du point $O$. En effet, jusqu'à l'instant $0,5+10=10,5$ il n'y a pas d'onde de retour en $b^{4}$ et les points de fonctionnement en $b^{4}$ antérieur à 10,5 , doivent être sur la même droite o'l' passant par le point initial $(q=0$, $\xi=0$ ). Donc $1,5_{b^{\circ}}$ et $1,5_{b^{\prime}}$ sont confondus avec l'intersection de la droite $\Phi^{\prime}$ issue de $1_{b^{\prime}}$ et de la droite $\rho^{\prime \prime \prime}$ issue de $\mathrm{O}$.

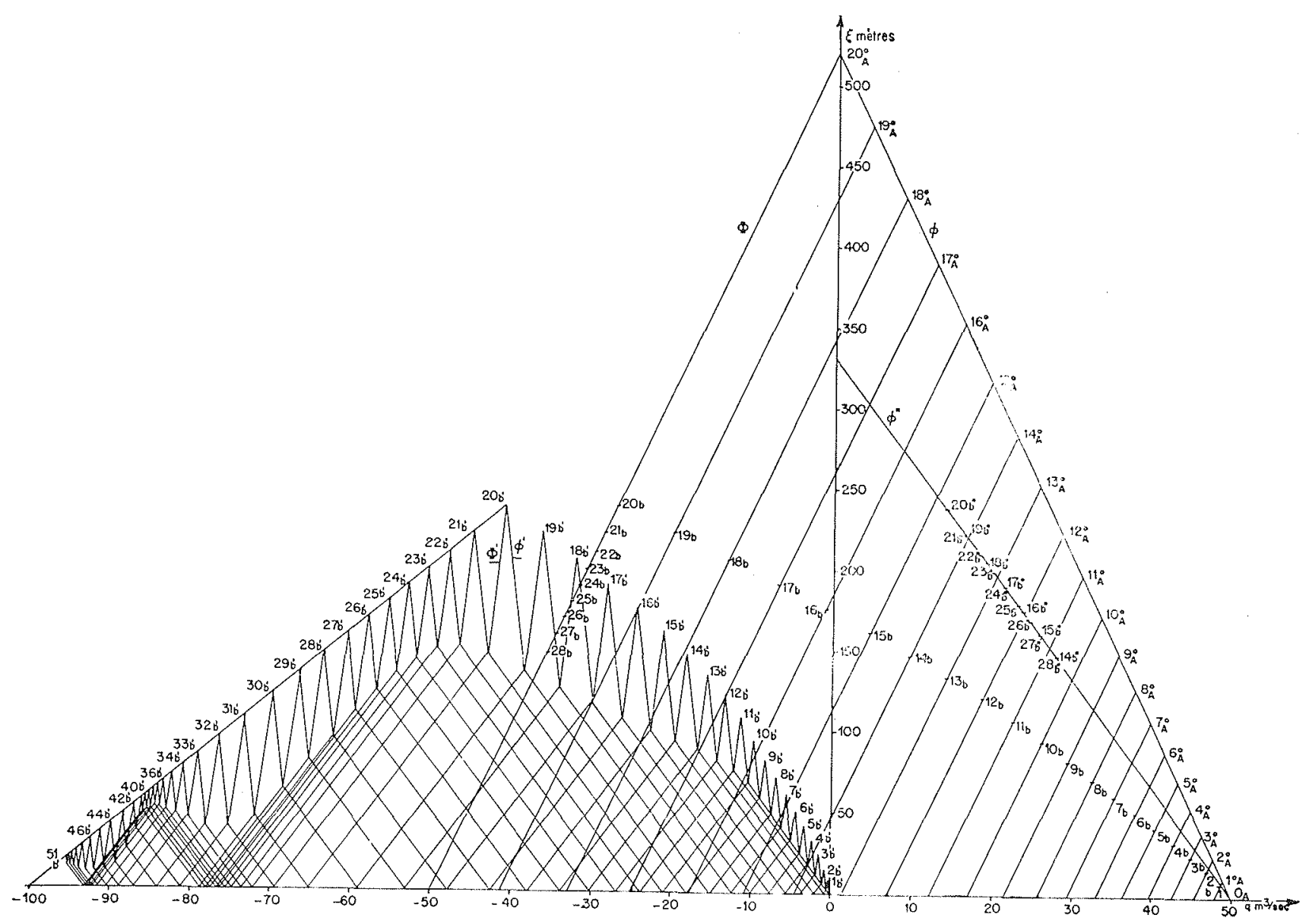

Fig. 20 
L'onde $f^{\prime}$ partie de $b^{3}$ en $1,5\left(1,5_{b^{*}}\right)$ arrive en $b^{\prime}$ en $2\left(2_{b^{\prime}}\right)$ : donc $2_{b^{\prime}}$ est sur la droite $\varphi^{\prime}$ issue de 1,5 .

On construirait de même :

$2,5 b^{3}$ sur la droite $\varphi^{\prime \prime}$ et sur la droite (I) issue de $2_{b^{\prime}}$.

$3_{b^{\prime}} \quad$ sur la droite $\varphi^{\prime}$ issue de $2,5_{b^{\prime \prime}}$.

$3,5 b^{3}$ sur la droite " $"$ " et sur la droite $\Phi$ issue de $3_{b}$, .

$11_{b^{\prime}}$ sur la droite $Q^{\prime}$ issue de $10.5 b^{2}$.

L'onde $F^{\prime \prime \prime}$ partie de $b^{i}$ en 1,5 arrive en $1,5+5=6,5$ en $b^{5}$ où la surpression est nulle, donc $6,5 \mathrm{~b}^{5}$ est sur la droite $\Phi^{\prime \prime \prime}$ issue de $1,5_{b^{\prime \prime}}$ et sur l'axe des débits.

L'onde $f^{\prime \prime \prime}$ partie de $b^{5}$ à l'instant $6,5\left(6,5_{b^{5}}\right)$ revient en $b^{4}$ à l'instant $6,5+5=11,5$, donc le point de fonctionnement $11,5 b^{4}$ confondu avec $11,5_{b^{\prime}}$ est situé sur la droite $o^{\prime \prime \prime}$ issue de $6,5_{b^{5}}$; il est, d'autre part, situé sur la droite $\Phi^{\prime}$ issue de $11_{b}$, ce qui le détermine.

De même 12,5 $b_{b^{\prime}}$ est sur la droite $\varphi^{\prime}$ issue de $12_{b}$, et sur la droite $q^{\prime \prime \prime}$ réfléchie sur $\mathrm{O} \xi$ par le point $2,5_{b}, \ldots$ etc..., d'où la construction.

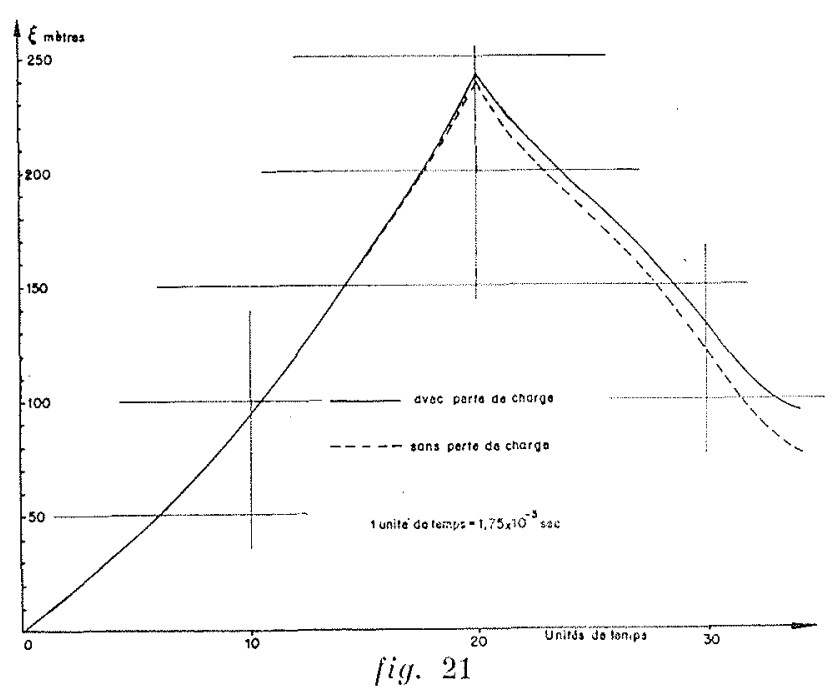

La figure 21 donne la courbe de surpression obtenue en fonction du temps. Le maximum atteint $239,50 \mathrm{~m}$. est très élevé, et bien supérieur à celui que l'on obtient dans les mêmes conditions, 150,60 m. (tab. 1) (1) lorsque l'étranglement débouche directement dans la chambre d'équilibre. Ce résultat ne doit pas surprendre car, si l'étranglement n'existaił pas, le tube de liaison seul fransmettrait au canal d'amenée une

(1) p. 469 . onde dont le maximum atreindraiñ $188 \mathrm{~m}$., toutes les autres données demeurant inchangées, comme il résulte de la construction graphique de la figure 22, dans laquelle l'unité de temps est prise

égale à $\theta^{\prime \prime \prime}=\frac{\mathrm{T}}{2}$.

Les constructions précédentes négligent la perte de charge dans l'étranglement. II est facile de tenir compte de cet élément: par exemple, si on suppose cette perte localisée dans la section terminale supérieure de l'étranglement, la construction devient celle de la figure 23 , chaque droite $\varphi^{\prime \prime \prime}$ étant maintenant solidaire de la parabole déformée des pertes de charge, suivant la méthode classique indiquée par M. BERGERON. La courbe de surpression (fig. 21) passe par un maximum de $244 \mathrm{~m}$., à peine supérieur, dans le cas actuel, à celui que l'on obtient en l'absence de pertes de charge.

\section{CHAPITRE VI \\ CONCLUSIONS}

$1^{\circ}$ L'onde de surpression à front vertical provoquée par une fermeture rigoureusement instantanée se transmet toujours au canal d'amenée, sauf dans le cas où le canal et la conduite débouchent directement dans une chambre d'équilibre de section infinie: par exemple, dans un cas particulier, si un étranglement permet la transmission d'une onde incidente dans la proportion de $90 \%$, un tube de liaison de section égale à celle du canal d'amenée permet la même transmission dans une proportion de $65 \%$, inférieure mais comparable à la précédente. Une fermeture rigoureusement instantanée n'intervient heureusement jamais dans la pratique.

$2^{\circ}$ Dans l'étude des manœuvres de fermeture brusque de très courte durée, nous avons envisagé des circonstances tout à fait anormales et fort heureusement irréalisables en pratique, puisqu'elles correspondraient à des surpressions dans la conduite supérieures au double de la pression statique: de telles mancuvres de fermeture brusque, de très courte durée, ne peuvent guère affecter, même accidentellement, qu'une petite fraction du débit maximum. La méthode graphique de $M$. BERCERON et la formule abrégée qui en résulte permettent de se rendre compte qu'en pratique les ondes transmises du fait de la présence de l'étranglement, 


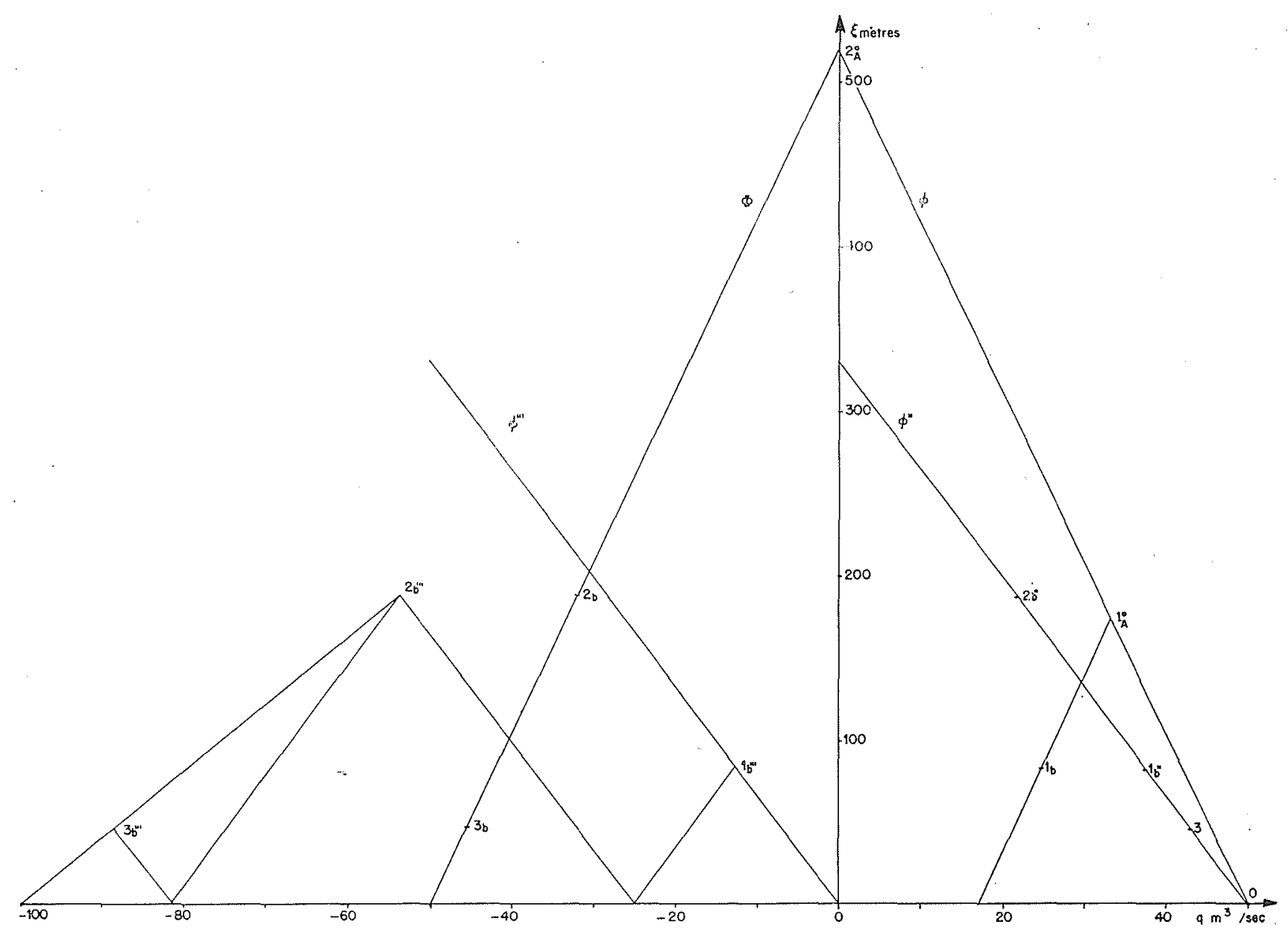

fig. 22

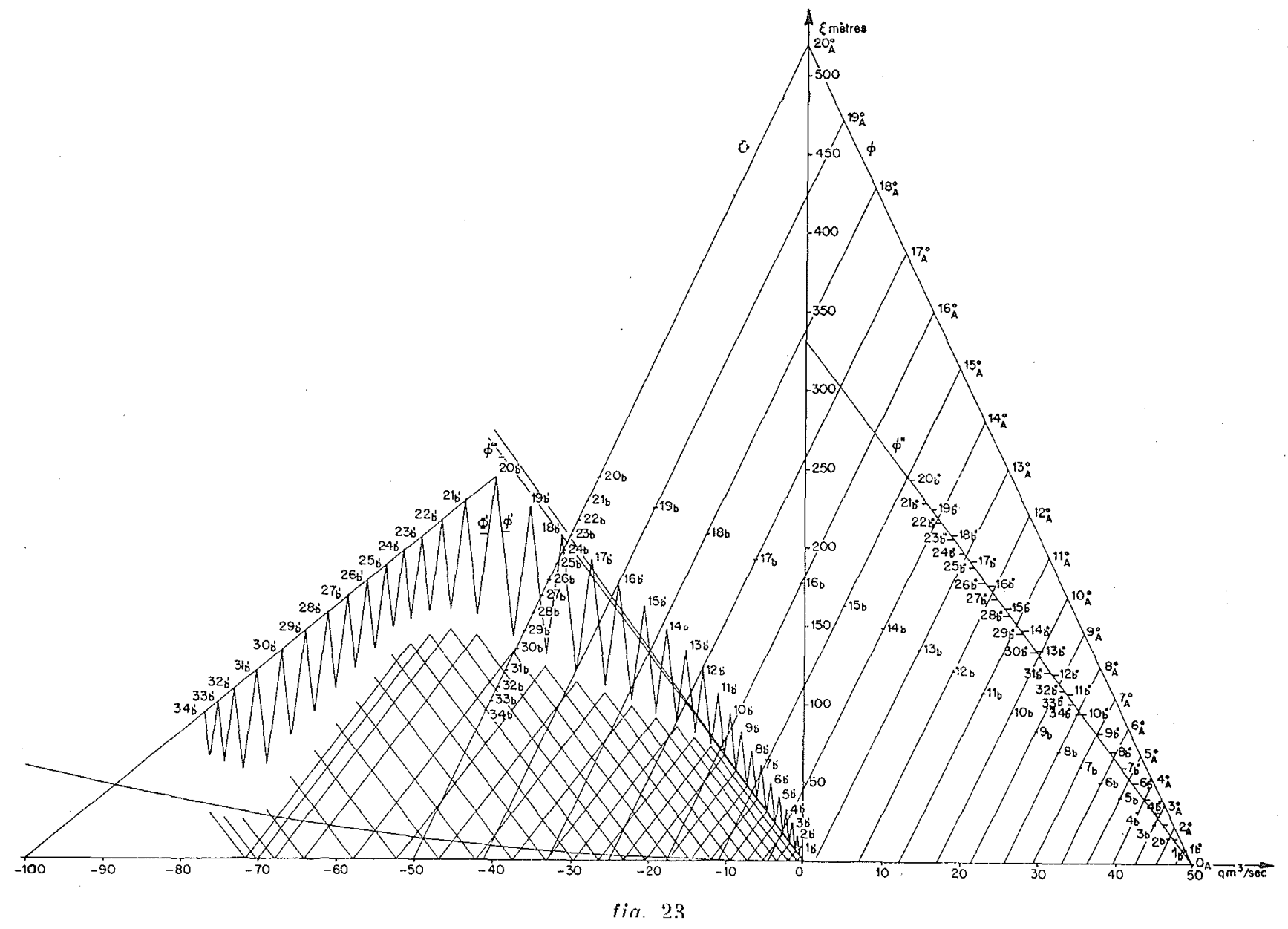


dans de telles circonstances, essentiellement accidentelles, ne peuvent atteindre des valeurs gênantes, et cela d'autant moins que l'étranglement est plus court: nous avons vu d'ailleurs que, dans de telles conditions anormales de fonctionnement, un simple tube de liaison, de section égale à celle du canal d'amenée et de quelques mètres de longueur, suffit, en l'absence de tout étranglement, à permettre la transmission partielle des surpressions.

$3^{\circ}$ Les mancuvres de fermeture lente $(T>\theta)$, seules utilisées en pratique, ne peuvent permettre à un étranglement de courte longueur de transmettre au canal d'amenée des ondes de pression appréciables: seule, la perte de charge à la traversée de l'étranglement intervient pour majorer la pression dans le canal, dans les con- ditions que considère le calcul classique des oscillations en masse.

$4^{\circ}$ Par contre, ces mancuvres de fermeture lente peuvent, qu'il y ait ou non un étranglement à la base de la chambre d'équilibre, créer une surpression notable dans le canal d'amenée, lorsque la liaison avec l'épanouissement de la chambre d'équilibre est assurée par une conduite de grande longueur, cas exceptionnel, que peuvent imposer toutefois les conditions locales comme dans l'exemple de l'usine des Brévières.

Nous retiendrons, en particulier, de ces diverses conclusions, qu'il est possible d'adopter le dispositif d'étranglement dans toutes les usines comportant une chambre d'équilibre, sans que cela puisse faire craindre des conséquences fâcheuses.

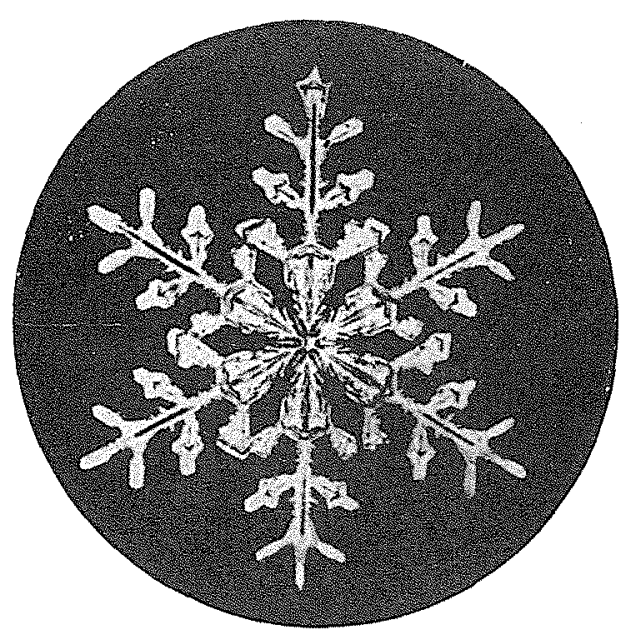

